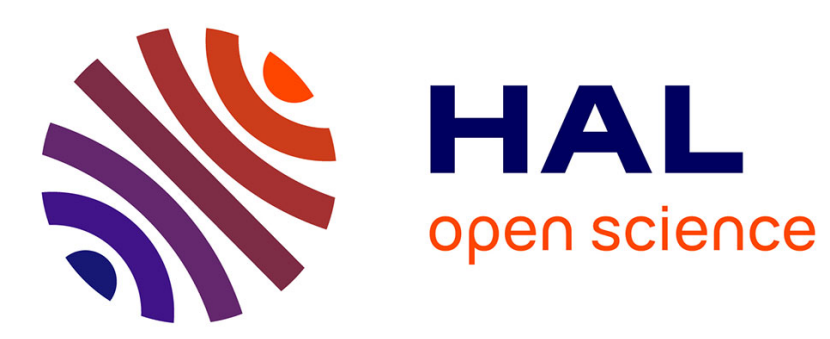

\title{
On the maximum time step in weakly compressible SPH
}

Damien Violeau, Agnès Leroy

\section{To cite this version:}

Damien Violeau, Agnès Leroy. On the maximum time step in weakly compressible SPH. Journal of Computational Physics, 2014, 256, pp.388-415. hal-00946833

\section{HAL Id: hal-00946833 https://hal.science/hal-00946833}

Submitted on 14 Feb 2014

HAL is a multi-disciplinary open access archive for the deposit and dissemination of scientific research documents, whether they are published or not. The documents may come from teaching and research institutions in France or abroad, or from public or private research centers.
L'archive ouverte pluridisciplinaire HAL, est destinée au dépôt et à la diffusion de documents scientifiques de niveau recherche, publiés ou non, émanant des établissements d'enseignement et de recherche français ou étrangers, des laboratoires publics ou privés. 


\title{
On the maximum time step in weakly compressible SPH
}

\author{
Damien Violeau (corresponding author), damien.violeau@edf.fr, \\ +33 (0)1 30877831 and Agnès Leroy, agnes.leroy@edf.fr \\ Laboratoire d'Hydraulique Saint-Venant, 6 quai Watier, 78400 Chatou, France
}

\begin{abstract}
In the SPH method for viscous fluids, the time step is subject to empirical stability criteria. We proceed to a stability analysis of the Weakly Compressible SPH equations using the von Neumann approach in arbitrary space dimension for unbounded flow. Considering the continuous SPH interpolant based on integrals, we obtain a theoretical stability criterion for the time step, depending on the kernel standard deviation, the speed of sound and the viscosity. The stability domain appears to be almost independent of the kernel choice for a given space discretization. Numerical tests show that the theory is very accurate, despite the approximations made. We then extend the theory in order to study the influence of the method used to compute the density, of the gradient and divergence SPH operators, of background pressure, of the model used for viscous forces and of a constant velocity gradient. The influence of time integration scheme is also studied, and proved to be prominent. All of the above theoretical developments give excellent agreement against numerical results. It is found that velocity gradients almost do not affect stability, provided some background pressure is used. Finally, the case of bounded flows is briefly addressed from numerical tests in three cases: a laminar Poiseuille flow in a pipe, a lid-driven cavity and the collapse of a water column on a wedge.
\end{abstract}

Keywords: SPH, numerical stability, weakly compressible, viscous flow, kernel

Fourier transform

\section{Introduction}

The Smoothed Particle Hydrodynamics (SPH) Lagrangian numerical method has been more and more used during the past years, thanks to its capability to model complex physical problems, in particular in the field of fluid mechanics. However, many of its numerical properties are still poorly known. In particular, the question of numerical stability has been treated for a long time, but there is no clear theory 
allowing the user to determine the optimal time step, i.e. the maximum time step allowed for keeping numerical stability of the solutions. Indeed, the explicit nature of standard SPH suggests that this method is conditionally stable, a statement which is confirmed by numerical evidence. In all the available SPH literature, the time step is chosen according to empirical laws based on the numerical speed of sound, the viscosity and the smoothing length, inspired from the mesh-based methods (see e.g. Morris et al., 1997). The purpose of the present work is to deduce the maximum time step from theoretical investigations. In the following we assume the reader to be familiar with SPH (for a broad discussion of this method, see Monaghan, 2005).

There are two approaches in studying SPH stability. The first one consists of keeping the discrete nature of the method in the analysis, the second one consists of approximating the discrete SPH interpolation with its continuous form based on the interpolating kernel. The latter can be performed in arbitrary dimension (see Balsara, 1995), but as an approximation it cannot handle all the numerical properties of SPH. The discrete approach is closer to the real SPH method but can hardly be conducted in arbitrary space dimension and with arbitrary neighbour particles. With this approach, the particles are usually assumed to be placed on a cartesian grid and only the closest neighbours are considered. Swegle et al. (1995) conducted a linear stability analysis of SPH for solids from this discrete point of view in one dimension, and determined a stability criterion based on the sign of the stress and kernel second derivative. Morris (1996) extended this work to arbitrary number of neighbour particles for fluid flow, including viscous effects. On the other hand, Balsara (1995) used the method of continuous operators to investigate stability of SPH for fluids in one dimension and studied the impact of kernel and artificial viscosity. The last two authors came to the conclusion that the Fourier transform of the kernel plays a major role in the stability properties of SPH, which was later confirmed by Robinson's $\mathrm{PhD}$ thesis (2009). However, none of these papers give an explicit criterion for the maximum allowed time step.

We concentrate here on SPH for viscous isothermal fluids, non self-gravitational flows, using the so-called Weakly-Compressible approach (WCSPH) proposed by Monaghan (1994) and widely used in theoretical and applied publications. We will chose usual forms of the discrete pressure gradient, viscous forces and velocity divergence and proceed to a von Neumann stability analysis. For this purpose, we will use the continuous approach instead of the discrete one, which allows us working in 
arbitrary dimension. In the major part of the paper we will focus on unbounded flows. In Section 2, the continuous SPH governing equations will be linearised from a reference state and a theoretical stability criterion for the time step will be deduced, then confirmed by numerical tests with an excellent accuracy for all tested kernels. We will confirm that the kernel Fourier transform is a key function in the stability properties of SPH. In Section 3 we will look at the effect of various model options. Indeed there are several approaches in SPH for predicting the evolution of density (continuity equation or density interpolation) as well as various forms of the pressure gradient (momentum-conserving and zero-order consistent forms, to mention the most two important ones). The pros and cons of these approaches are discussed by many authors, in particular by Vila (1999) and Price (2012). In the present study, we will see that the choice for pressure gradient modelling and density computation have no influence on our results. We will also study the effect time integration scheme, comparing frist-order and second-order (leapfrog) schemes. We will see that the time scheme has a major effect on the stability criterion, as well as the model for viscous forces and the use of background pressure. Finally, we will try to extend these results to real flows from numerical tests, including the effect of solid walls.

\section{Linear stability analysis of continuous SPH 2.1. SPH continuous governing equations}

We start by defining our notations and tools for the purpose of the present study. For more detail about SPH, the reader can refer to Monaghan (2005) or Violeau (2012).

The most frequently used gradient (hereafter denoted by $\mathbf{G}$ ) and divergence $(D)$ operators are derived from the following continuous SPH approximation of an arbitrary scalar field $A$ :

$$
\begin{aligned}
A(\mathbf{r}) & =\int_{\Omega} A\left(\mathbf{r}^{\prime}\right) \delta\left(\mathbf{r}-\mathbf{r}^{\prime}\right) d \mathbf{r}^{\prime} \\
& =\int_{\Omega} A\left(\mathbf{r}^{\prime}\right) w\left(\left|\mathbf{r}-\mathbf{r}^{\prime}\right|\right) d \mathbf{r}^{\prime}+O\left(h^{2}\right)
\end{aligned}
$$

where $\delta$ is the Dirac distribution and $w$ the SPH kernel, here assumed isotropic, i.e. to depend on the distance $\left|\mathbf{r}-\mathbf{r}^{\prime}\right|$ only. For practical applications, the kernel is often compactly supported, with smoothing length $h$, although the Gaussian kernel (with infinite support) is often used to perform analytical developments. The order

of approximation $O\left(h^{2}\right)$ in (1) is a consequence of the kernel isotropy, as well as the normalising condition, i.e. the integral of $w$ on the fluid domain $\Omega$ is equal to 1 . This 
is only true if the point of interest $\mathbf{r}$ is far enough from the boundaries, which will be assumed hereafter (except in Section 3.3). In most SPH papers, the smoothing length is used as space discretization scale. However, Dehnen and Aly (2012) recently pointed out that a better choice should be the kernel standard deviation $\sigma$, defined by

$$
\sigma^{2} \doteqdot \frac{1}{n} \int_{\Omega} \tilde{r}^{2} w(\tilde{r}) d \tilde{\mathbf{r}}
$$

It has the advantage to be finite even with the Gaussian kernel, and to give a correct measure of the number of particles in the kernel support, irrespective of the kernel choice. The values of the ratio $\sigma / h$ for various kernels are given in Table 1 . In the following, we will use $\sigma$ in place of $h$ (see Section 2.4).

Applying the approximation (1) to $\nabla A$ gives

$$
\begin{aligned}
\nabla A(\mathbf{r}) & \approx \int_{\Omega} \nabla A\left(\mathbf{r}^{\prime}\right) w\left(\left|\mathbf{r}-\mathbf{r}^{\prime}\right|\right) d \mathbf{r}^{\prime} \\
& =\int_{\partial \Omega} A\left(\mathbf{r}^{\prime}\right) w\left(\left|\mathbf{r}-\mathbf{r}^{\prime}\right|\right) \mathbf{n}\left(\mathbf{r}^{\prime}\right) d \Gamma\left(\mathbf{r}^{\prime}\right)-\int_{\Omega} A\left(\mathbf{r}^{\prime}\right) \nabla_{\mathbf{r}^{\prime}} w\left(\left|\mathbf{r}-\mathbf{r}^{\prime}\right|\right) d \mathbf{r}^{\prime}
\end{aligned}
$$

where $\mathbf{n}\left(\mathbf{r}^{\prime}\right)$ is the outward normal vector to the boundary $\partial \Omega$ at the point $\mathbf{r}^{\prime}$. Being far from the boundary, we can remove the first integral in the case of compactly supported kernel, while the second one can be modified using the antisymmetry of the kernel gradient, i.e. $\nabla_{\mathbf{r}^{\prime}} w\left(\left|\mathbf{r}-\mathbf{r}^{\prime}\right|\right)=-\nabla_{\mathbf{r}} w\left(\left|\mathbf{r}-\mathbf{r}^{\prime}\right|\right)$, which is a consequence of the kernel symmetry $\left(\nabla_{\mathbf{r}}\right.$ denotes the gradient with respect to the components of $\mathbf{r}$, i.e. $\partial / \partial \mathbf{r})$. It should be underlined, however, that the Gaussian kernel having an infinite support, the boundary integral can only be removed in the ideal case of an 'infinite' fluid domain. The latter assumptions give a first SPH continuous gradient operator:

$$
\mathbf{G}\{A\}(\mathbf{r}) \doteqdot \int_{\Omega} A\left(\mathbf{r}^{\prime}\right) \nabla_{\mathbf{r}} w\left(\left|\mathbf{r}-\mathbf{r}^{\prime}\right|\right) d \mathbf{r}^{\prime} \approx \nabla A(\mathbf{r})
$$

On similar grounds, a continuous divergence SPH operator is defined for an arbitrary vector field $\mathbf{A}$ as

$$
D\{\mathbf{A}\}(\mathbf{r}) \doteqdot \int_{\Omega} \mathbf{A}\left(\mathbf{r}^{\prime}\right) \cdot \nabla_{\mathbf{r}} w\left(\left|\mathbf{r}-\mathbf{r}^{\prime}\right|\right) d \mathbf{r}^{\prime} \approx \nabla \cdot \mathbf{A}(\mathbf{r})
$$

We now define the following modified continuous operators:

$$
\begin{aligned}
& \mathbf{G}^{+}\{A\} \doteqdot \rho \mathbf{G}\left\{\frac{A}{\rho}\right\}+\frac{A}{\rho} \mathbf{G}\{\rho\} \\
& D^{-}(\mathbf{A}) \doteqdot \frac{1}{\rho} D\{\rho \mathbf{A}\}-\frac{\mathbf{A}}{\rho} \cdot \mathbf{G}\{\rho\}
\end{aligned}
$$


where $\rho$ is the fluid density field, giving, from (4) and (5):

$$
\begin{aligned}
& \mathbf{G}^{+}\{A\}(\mathbf{r})=\int_{\Omega} \frac{\rho\left(\mathbf{r}^{\prime}\right)^{2} A(\mathbf{r})+\rho(\mathbf{r})^{2} A\left(\mathbf{r}^{\prime}\right)}{\rho(\mathbf{r}) \rho\left(\mathbf{r}^{\prime}\right)} \nabla_{\mathbf{r}} w\left(\left|\mathbf{r}-\mathbf{r}^{\prime}\right|\right) d \mathbf{r}^{\prime} \\
& D^{-}\{\mathbf{A}\}(\mathbf{r})=-\int_{\Omega} \frac{\rho\left(\mathbf{r}^{\prime}\right)}{\rho(\mathbf{r})}\left[\mathbf{A}(\mathbf{r})-\mathbf{A}\left(\mathbf{r}^{\prime}\right)\right] \cdot \nabla_{\mathbf{r}} w\left(\left|\mathbf{r}-\mathbf{r}^{\prime}\right|\right) d \mathbf{r}^{\prime}
\end{aligned}
$$

Note that the relations (6) are exact when applied to the standard continuous differential operators gradient (i.e. $\nabla$ in place of $\mathbf{G})$ and divergence $(\nabla \cdot$ in place of $D)$. Thus, $\mathbf{G}^{+}$and $D^{-}$are relevant approximations of $\nabla$ and $\nabla \cdot$, respectively. The 'plus' and 'minus' superscripts suggest that these SPH operators were built with plus and minus signs respectively, while other possibilities exist, as we will see in Section 3.1. Now, the traditional SPH discrete differential operators are written as discrete sums involving fluid particles. They can be obtained from (7) as

$$
\begin{aligned}
& \mathbf{G}_{a}^{+}\left\{A_{b}\right\} \doteqdot \rho_{a} \sum_{b} m_{b}\left(\frac{A_{a}}{\rho_{a}^{2}}+\frac{A_{b}}{\rho_{b}^{2}}\right) \nabla w_{a b} \\
& D_{a}^{-}\left\{\mathbf{A}_{b}\right\} \doteqdot-\frac{1}{\rho_{a}} \sum_{b} m_{b} \mathbf{A}_{a b} \cdot \nabla w_{a b}
\end{aligned}
$$

where the subscripts refer to particles, e.g. $\rho_{b} \doteqdot \rho\left(\mathbf{r}_{b}\right), \mathbf{r}_{b}$ being the position of particle $b$ at a certain time. We also define the particle mass $m_{b}$, as well as $A_{a b} \doteqdot$ $A_{a}-A_{b}, \mathbf{A}_{a b} \doteqdot \mathbf{A}_{a}-\mathbf{A}_{b}$ and $\nabla w_{a b} \doteqdot \nabla_{\mathbf{r}_{a}} w\left(\left|\mathbf{r}_{a}-\mathbf{r}_{b}\right|\right)$, according to the usual notations in the SPH literature. Note that the divergence operator $D_{a}^{-}$is zeroorder consistent, i.e. vanishes for constant fields (Bonet and Lok (1999) also show how to improve consistency up to the first order by using renormalisation matrices). On the other hand, the present discrete gradient $\mathbf{G}_{a}^{+}$does not fulfil this property. However, it is mostly used in the SPH literature because it satisfies linear momentum conservation, as resulting from a variational principle (see e.g. Monaghan, 1992). For this reason, this form of the SPH gradient is highly recommended (Price, 2012).

The Laplacian operator can also be written in discrete and continuous forms. We first consider the model proposed by Morris et al. (1997), widely used in the SPH literature for modelling viscous forces:

$$
\begin{aligned}
& \mathbf{L}\{B, \mathbf{A}\}(\mathbf{r}) \\
\doteqdot & \int_{\Omega}\left[B(\mathbf{r})+B\left(\mathbf{r}^{\prime}\right)\right]\left[\mathbf{A}(\mathbf{r})-\mathbf{A}\left(\mathbf{r}^{\prime}\right)\right] \frac{\mathbf{r}-\mathbf{r}^{\prime}}{\left|\mathbf{r}-\mathbf{r}^{\prime}\right|^{2}} \cdot \nabla_{\mathbf{r}} w\left(\left|\mathbf{r}-\mathbf{r}^{\prime}\right|\right) d \mathbf{r}^{\prime} \\
\approx & \nabla \cdot[B(\mathbf{r}) \nabla \mathbf{A}(\mathbf{r})]
\end{aligned}
$$

It is actually more general than the Laplacian, rather a diffusion-like term based on a diffusion coefficient $B(\mathbf{r})$ which can vary in space, with the following discrete 
equivalent form:

$$
\mathbf{L}_{a}\left\{B_{b}, \mathbf{A}_{b}\right\} \doteqdot \sum_{b} V_{b}\left(B_{a}+B_{b}\right) \mathbf{A}_{a b} \frac{\mathbf{r}_{a b}}{r_{a b}^{2}} \cdot \nabla w_{a b}
$$

where $V_{b} \doteqdot m_{b} / \rho_{b}$ is the particle volume, $r_{a b} \doteqdot\left|\mathbf{r}_{a b}\right|$ and $\mathbf{r}_{a b} \doteqdot \mathbf{r}_{a}-\mathbf{r}_{b}$. Schwaiger (2008) and Fatehi and Manzari (2011) proposed second-order consistent Laplacian operators for SPH, but they are significantly more complex and will not be considered in the present work.

The numerical error done when approximating the continuous operators (7) with the discrete ones (8) depends on the particle distribution in the fluid, and was investigated in Quinlan et al. (2006) and Amicarelli et al. (2011). In the following, we will only consider the continuous forms (7) and (9), which will make our stability analysis easier. As pointed out by Balsara (1995), the error done by doing so is so small that it hardly affects the results of stability analysis, and we will confirm this statement later on from numerical tests. Thus, the role of Lagrangian particles $b$ will be played by the dummy argument $\mathbf{r}^{\prime}$ in the integrals, i.e. $A\left(\mathbf{r}^{\prime}\right)$ stands for $A_{b}$ in the continuum limit.

We restrict our work to viscous isothermal fluid modelling based on the traditional Weakly Compressible SPH approach (here referred to as WCSPH, see Monaghan, 1994 and Morris et al., 1997). We also neglect self-gravitation. We will thus consider the following continuous SPH forms of the Navier-Stokes momentum equation and of the continuity equation:

$$
\begin{aligned}
& \dot{\mathbf{u}}(\mathbf{r})=-\frac{1}{\rho(\mathbf{r})} \mathbf{G}^{+}\{p\}(\mathbf{r})+\frac{1}{\rho(\mathbf{r})} \mathbf{L}\{\mu, \mathbf{u}\}(\mathbf{r}) \\
& \dot{\rho}(\mathbf{r})=-\rho(\mathbf{r}) D^{-}\{\mathbf{u}\}(\mathbf{r})
\end{aligned}
$$

where $p$ and $\mathbf{u}$ are the pressure and velocity field, respectively, while $\mu$ is the dynamic viscosity and the dots denote Lagrangian time derivatives. The pressure is computed from the following equation of state (Monaghan, 1994):

$$
p(\mathbf{r})=\frac{\rho_{0} c_{0}^{2}}{\gamma}\left(\frac{\rho(\mathbf{r})^{\gamma}}{\rho_{0}^{\gamma}}-1\right)
$$

$c_{0}$ being the numerical sound speed at the reference density $\rho_{0}$, and $\gamma=7$ for water.

\subsection{Linearisation}

Consider a reference state corresponding to homogeneous conditions, i.e. $\mathbf{u}(\mathbf{r})=\mathbf{c s t}$ and $\rho(\mathbf{r})=\rho_{0}$ (which is a solution to the Navier-Stokes equations and to the $\mathrm{SPH}$ continuous system (11)). Suppose the positions, velocities and densities are now 
modified by small arbitrary amounts $\delta \mathbf{r}, \delta \rho(\mathbf{r})$ and $\delta \mathbf{u}(\mathbf{r})$, respectively. Then, it is demonstrated in Appendix 1 that the fluid acceleration and density variation are modified by $\delta \dot{\mathbf{u}}(\mathbf{r})$ and $\delta \dot{\rho}(\mathbf{r})$ as follows:

$$
\begin{aligned}
\delta \dot{\mathbf{u}}(\mathbf{r})= & -\frac{c_{0}^{2}}{\rho_{0}} \int_{\Omega}\left[\delta \rho(\mathbf{r})+\delta \rho\left(\mathbf{r}^{\prime}\right)\right] \nabla_{\mathbf{r}} w\left(\left|\mathbf{r}-\mathbf{r}^{\prime}\right|\right) d \mathbf{r}^{\prime} \\
& +2 \nu \int_{\Omega}\left[\delta \mathbf{u}(\mathbf{r})-\delta \mathbf{u}\left(\mathbf{r}^{\prime}\right)\right] \frac{\mathbf{r}-\mathbf{r}^{\prime}}{\left|\mathbf{r}-\mathbf{r}^{\prime}\right|^{2}} \cdot \nabla_{\mathbf{r}} w\left(\left|\mathbf{r}-\mathbf{r}^{\prime}\right|\right) d \mathbf{r}^{\prime} \\
\delta \dot{\rho}(\mathbf{r})= & \rho_{0} \int_{\Omega}\left[\delta \mathbf{u}(\mathbf{r})-\delta \mathbf{u}\left(\mathbf{r}^{\prime}\right)\right] \cdot \nabla_{\mathbf{r}} w\left(\left|\mathbf{r}-\mathbf{r}^{\prime}\right|\right) d \mathbf{r}^{\prime}
\end{aligned}
$$

with $\nu \doteqdot \mu / \rho_{0}$ the kinematic viscosity. We now consider the case of space-periodic fluctuations:

$$
\begin{gathered}
\delta \mathbf{u}=c_{0} \mathbf{U}(t) \exp (-i \mathbf{K} \cdot \mathbf{r}) \\
\delta \mathbf{r}=\sigma \mathbf{R}(t) \exp (-i \mathbf{K} \cdot \mathbf{r}) \\
\delta \rho=\rho_{0} R(t) \exp (-i \mathbf{K} \cdot \mathbf{r})
\end{gathered}
$$

where $\mathbf{U}(t), \mathbf{R}(t)$ and $R(t)$ are arbitrary dimensionless functions of time and $\mathbf{K}$ a wavevector (the fluctuations of particle positions $\delta \mathbf{r}$ are not used in the present calculations but will be useful in Section 3.1). Recall $\sigma$ is the kernel standard deviation as defined by eqn (2). By making the variable change $\tilde{\mathbf{r}}=\mathbf{r}^{\prime}-\mathbf{r}$ (hence $\nabla_{\mathbf{r}}=-\nabla_{\tilde{\mathbf{r}}}$ and $\left.d \mathbf{r}^{\prime}=d \tilde{\mathbf{r}}\right)$ we obtain

$$
\begin{aligned}
\delta \dot{\mathbf{u}}(\mathbf{r}) \exp (i \mathbf{K} \cdot \mathbf{r})= & c_{0}^{2} R(t) \int_{\Omega}[1+\exp (-i \mathbf{K} \cdot \tilde{\mathbf{r}})] \nabla_{\tilde{\mathbf{r}}} w(\tilde{r}) d \tilde{\mathbf{r}} \\
& +2 \nu c_{0}\left[\int_{\Omega}[1-\exp (-i \mathbf{K} \cdot \tilde{\mathbf{r}})] \frac{\tilde{\mathbf{r}}}{\tilde{r}} \cdot \nabla_{\tilde{\mathbf{r}}} w(\tilde{r}) d \tilde{\mathbf{r}}\right] \mathbf{U}(t)
\end{aligned}
$$

where $\tilde{r} \doteqdot|\tilde{\mathbf{r}}|$. The integral of $\nabla_{\tilde{\mathbf{r}}} w(\tilde{r})$ vanishes because of Gauss' theorem and the compactly supported kernel. We now use the following property of the multidimensional Fourier transform (hereafter denoted by a hat):

$$
\sigma \widehat{\nabla_{\tilde{\mathbf{r}}} w}(\mathbf{K})=i \widehat{w}\left(K^{*}\right) \mathbf{K}^{*}
$$

Where $\mathbf{K}^{*} \doteqdot \sigma \mathbf{K}$ is the dimensionless wavevector and $K^{*} \doteqdot\left|\mathbf{K}^{*}\right|$ the dimensionless wavenumber. $\widehat{w}$ is a function of the wavenumber (i.e. the wavevector length) because of the kernel isotropy property (the Fourier transform of a radial function is another radial function) and because we assumed the boundaries to be removed far away from the point of interest. The use of $\sigma$ as scaling distance in place of $h$ makes the kernel Fourier transform almost independent on the kernel choice, especially at small 
wavenumbers, as pointed out by Dehnen and Aly (2012) (see also Appendix 2). We get

$$
\delta \dot{\mathbf{u}}(\mathbf{r}) \exp (i \mathbf{K} \cdot \mathbf{r})=\frac{i c_{0}^{2}}{\sigma} \widehat{w}\left(K^{*}\right) R(t) \mathbf{K}^{*}-\frac{\nu c_{0}}{\sigma^{2}} F_{2}\left(K^{*}\right) \mathbf{U}(t)
$$

where we have defined

$$
F_{2}\left(K^{*}\right) \doteqdot 2 \sigma^{2} \int_{\Omega}[\exp (-i \mathbf{K} \cdot \tilde{\mathbf{r}})-1] \frac{\tilde{\mathbf{r}}}{\tilde{r}^{2}} \cdot \nabla_{\tilde{\mathbf{r}}} w(\tilde{r}) d \tilde{\mathbf{r}}
$$

which is again a function of the wavenumber, regardless of the wavevector direction for kernel isotropy reasons.

Similarly, for the linearized continuity equation (84):

$$
\begin{aligned}
& \delta \dot{\rho}(\mathbf{r}) \exp (i \mathbf{K} \cdot \mathbf{r}) \\
= & -\rho_{0} c_{0} \mathbf{U}(t) \cdot \int_{\Omega}[1-\exp (-i \mathbf{K} \cdot \tilde{\mathbf{r}})] \nabla_{\tilde{\mathbf{r}}} w(\tilde{r}) d \tilde{\mathbf{r}} \\
= & \frac{i \rho_{0} c_{0}}{\sigma} \widehat{w}\left(K^{*}\right) \mathbf{K}^{*} \cdot \mathbf{U}(t)
\end{aligned}
$$

Gathering (17) and (19), and writing the time derivative of the particle positions as $\dot{\mathbf{r}}=\mathbf{u}$, we obtain the following system:

$$
\begin{aligned}
\delta \dot{\mathbf{u}}(\mathbf{r}) \exp (i \mathbf{K} \cdot \mathbf{r}) & =\frac{i c_{0}^{2}}{\sigma} \widehat{w}\left(K^{*}\right) R(t) \mathbf{K}^{*}-\frac{\nu c_{0}}{\sigma^{2}} F_{2}\left(K^{*}\right) \mathbf{U}(t) \\
\delta \dot{\mathbf{r}} \exp (i \mathbf{K} \cdot \mathbf{r}) & =c_{0} \mathbf{U}(t) \\
\delta \dot{\rho}(\mathbf{r}) \exp (i \mathbf{K} \cdot \mathbf{r}) & =\frac{i \rho_{0} c_{0}}{\sigma} \widehat{w}\left(K^{*}\right) \mathbf{K}^{*} \cdot \mathbf{U}(t)
\end{aligned}
$$

\subsection{Time integration scheme}

In order to analyse the behaviour of the solutions to the system (20), we must now choose a time integration scheme. So far, we have not considered this aspect, which will be proved to be very important in the following. Some authors (e.g. Morris, 1996; Antuono et al., 2012) conducted SPH stability analysis considering the time as a continuous variable. This idea is incorrect, as we will see. We will first concentrate on first-order schemes, i.e.

$$
\dot{A}(\mathbf{r}) \approx \frac{A\left(\mathbf{r}^{m+1}\right)-A\left(\mathbf{r}^{m}\right)}{\delta t}
$$

where the superscripts $m$ denote the time iteration and $\delta t$ the time step (a secondorder time marching scheme will be investigated in Section 3.2). We first investigate a sequential scheme, i.e. evaluating each of the right-hand sides of the system (20) using the values computed at the stage immediately before, i.e. using updated velocities 
to compute new positions and densities. Thus, for each particle we prescribe

$$
\begin{aligned}
& \frac{\mathbf{u}\left(t^{m+1}\right)-\mathbf{u}\left(t^{m}\right)}{\delta t}=\mathbf{F}\left(t^{m}\right) \\
& \frac{\mathbf{r}\left(t^{m+1}\right)-\mathbf{r}\left(t^{m}\right)}{\delta t}=\mathbf{u}\left(t^{m+1}\right) \\
& \frac{\rho\left(t^{m+1}\right)-\rho\left(t^{m}\right)}{\delta t}=D\left(t^{m+1}\right)
\end{aligned}
$$

where $\mathbf{F}$ denotes the force experienced by a particle per unit mass (right-hand side of the first line in eqn (11)), and $D$ the term $-\rho(\mathbf{r}) D^{-}\{\mathbf{u}\}(\mathbf{r})$ in eqn (11). Applying this scheme to model the derivatives in the system (20), we get

$$
\begin{aligned}
& \frac{\mathbf{U}\left(t^{m+1}\right)-\mathbf{U}\left(t^{m}\right)}{\delta t}=\frac{i c_{0}}{\sigma} \widehat{w}\left(K^{*}\right) R\left(t^{m}\right) \mathbf{K}^{*}-\frac{\nu}{\sigma^{2}} F_{2}\left(K^{*}\right) \mathbf{U}\left(t^{m}\right) \\
& \frac{\mathbf{R}\left(t^{m+1}\right)-\mathbf{R}\left(t^{m}\right)}{\delta t}=\frac{c_{0}}{\sigma} \mathbf{U}\left(t^{m+1}\right) \\
& \frac{R\left(t^{m+1}\right)-R\left(t^{m}\right)}{\delta t}=\frac{i c_{0}}{\sigma} \widehat{w}\left(K^{*}\right) \mathbf{K}^{*} \cdot \mathbf{U}\left(t^{m+1}\right)
\end{aligned}
$$

We will now seek for a wave-like solution, which amounts to looking for the unknown time-dependent functions as periodic functions:

$$
\begin{aligned}
& \mathbf{U}(t)=\mathbf{U}_{0} \exp (i \omega t) \\
& \mathbf{R}(t)=\mathbf{R}_{0} \exp (i \omega t) \\
& R(t)=R_{0} \exp (i \omega t)
\end{aligned}
$$

$\omega$ being an angular frequency. (23) now reads

$$
\begin{aligned}
\frac{\chi-1}{\delta t} \mathbf{U}_{0} & =\frac{i c_{0}}{\sigma} \widehat{w}\left(K^{*}\right) R_{0} \mathbf{K}^{*}-\frac{\nu}{\sigma^{2}} F_{2}\left(K^{*}\right) \mathbf{U}_{0} \\
\frac{\chi-1}{\delta t} \mathbf{R}_{0} & =\chi \frac{c_{0}}{\sigma} \mathbf{U}_{0} \\
\frac{\chi-1}{\delta t} R_{0} & =\chi \frac{i c_{0}}{\sigma} \widehat{w}\left(K^{*}\right) \mathbf{K}^{*} \cdot \mathbf{U}_{0}
\end{aligned}
$$

where $\chi \doteqdot \exp (i \omega \delta t)$ is the wave amplification factor. In other words, the numerical wave will be multiplied by the complex number $\chi$ at each iteration, thus a stability condition is $|\chi| \leqslant 1$. From (25), we find

$$
\left[\chi-1+C_{\nu} F_{2}\left(K^{*}\right)\right](\chi-1) K^{* 2} \mathbf{U}_{0}=-\chi C^{2} F_{1}\left(K^{*}\right)\left(\mathbf{K}^{*} \otimes \mathbf{K}^{*}\right) \mathbf{U}_{0}
$$

where we have used the following notation:

$$
F_{1}\left(K^{*}\right) \doteqdot\left[K^{*} \widehat{w}\left(K^{*}\right)\right]^{2}
$$


We also define the CFL (Courant-Friedrichs-Lewy) number $C$ and a viscous time step $C_{\nu}$ :

$$
\begin{gathered}
C \doteqdot \frac{c_{0} \delta t}{\sigma} \\
C_{\nu} \doteqdot \frac{\nu \delta t}{\sigma^{2}}=\frac{C}{R e_{0}}
\end{gathered}
$$

In the last equality, we defined the numerical Reynolds number as

$$
R e_{0} \doteqdot \frac{c_{0} \sigma}{\nu}
$$

The eigenvalues of the matrix $\mathbf{K}^{*} \otimes \mathbf{K}^{*}$ are 0 and $K^{* 2}$. From (26), the last one gives

$$
\chi^{2}-\left(2-A_{1}-A_{2}\right) \chi+1-A_{2}=0
$$

with, by definition:

$$
\begin{aligned}
& A_{1} \doteqdot C^{2} F_{1}\left(K^{*}\right) \\
& A_{2} \doteqdot C_{\nu} F_{2}\left(K^{*}\right)
\end{aligned}
$$

Note that a similar form of eqn (30) was obtained by Swegle et al. (1995) (their equation (A.42)). However, these authors worked with a discrete approach and only in dimension $n=1$. They did not care about the value of the time step, but deduce a necessary condition for numerical 'tensile' stability which basically states that the second derivative of the kernel for the smaller neighbouring particle distance should be positive (for a fluid, where normal stresses are negative), i.e. $w^{\prime \prime}(\delta r)$, where $\delta r$ is the particle spacing. This criterion, however, was severely criticized in Robinson's PhD thesis (2009) and more recently by Dehnen and Aly (2012). In the present theory, the equivalent of this condition is the positivity of $F_{1}\left(K^{*}\right)$, which is ensured by the definition (27). It is normal that the continuous approach proposed here cannot lead to a criterion like in Swegle et al.'s paper, where the discrete approach is the key tool. In the following, we will assume the function $F_{2}$ to be positive as well, which is true for all usual SPH kernels (see Section 2.4 and Appendix 3). Thus $A_{1}$ and $A_{2}$ are positive.

Calculating the roots $\chi$ of the second-order polynomial (30) leads to the following condition for $|\chi|$ to be less than unity:

$$
A_{1}+2 A_{2} \leqslant 4
$$


On the other hand, from (26) the zero eigenvalue of $\mathbf{K}^{*} \otimes \mathbf{K}^{*}$ gives $\chi=1$ (leading to stability) or $\chi=1-A_{2}$. The latter preserves stability provided $A_{2} \leqslant 2$, which is true under (32). Finally, the only stability condition which remains is (32), which should be satisfied for all values of $K^{*}$, giving the following stability condition:

$$
C \leqslant \sqrt{2 \min _{K^{*}} \frac{2-C_{\nu} F_{2}\left(K^{*}\right)}{F_{1}\left(K^{*}\right)}}
$$

The latter condition will now be analysed for various kernels and compared against numerical tests.

\subsection{Effect of the kernel choice}

The importance of the Fourier transform of the SPH kernel on numerical stability was already pointed out by Balsara (1995) for one-dimensional flow and more generally by Morris (1996) and Robinson (2009). From the definition (27) it can be seen that knowing $\widehat{w}\left(K^{*}\right)$ is enough to compute $F_{1}\left(K^{*}\right)$. Besides, from the definition (18), it is easy to see that the function $F_{2}\left(K^{*}\right)$ depends on $\widehat{w}\left(K^{*}\right)$ through the following relation, demonstrated in Appendix 2:

$$
F_{2}^{\prime}\left(K^{*}\right)=2 K^{*} \widehat{w}\left(K^{*}\right)=2 \sqrt{F_{1}\left(K^{*}\right)}
$$

with the boundary condition $F_{2}(0)=0$.

Let us now examine several usual kernels (see e.g. Monaghan, 1985; Wendland, 1995). Generally speaking, we may write

$$
\begin{gathered}
w(\tilde{r})=\frac{\alpha_{n}}{\tilde{h}^{n}} f(q) \\
q \doteqdot \frac{\tilde{r}}{\tilde{h}}
\end{gathered}
$$

where $n$ is the space dimension and $\alpha_{n}$ a normalising factor, $\tilde{h}$ being an appropriate scale. For all the kernels presented below, the normalizing constants $\alpha_{n}$ and the ratios $\sigma / h$ are given in Table 1. Following Dehnen and Aly (2012), we use $\tilde{h}=h=\sigma$ to define the Gaussian kernel by

$$
f(q)=\exp \left(-\frac{q^{2}}{2}\right)
$$

Its Fourier transform is given by

$$
\widehat{w}\left(K^{*}\right)=\exp \left(-\frac{K^{* 2}}{2}\right)
$$


and thus

$$
\begin{aligned}
& F_{1}\left(K^{*}\right)=K^{* 2} \exp \left(-K^{* 2}\right) \\
& F_{2}\left(K^{*}\right)=2\left[1-\exp \left(-\frac{K^{* 2}}{2}\right)\right]
\end{aligned}
$$

Still from Dehnen and Aly (2012), the other kernels defined below are based on the choice $\tilde{h}=H$, defined as the kernel support size (so that $q$ belongs to $[0,1]$ ). With this convention, the Wendland ${ }^{1} 5$ th order kernel reads

$$
f(q)=(1-q)^{4}(1+4 q)
$$

with $H / h=2$. Finally, we will consider the B-Spline kernels of orders 3,4 and 5 . The 3rd-order is defined by

$$
f(q)=(1-q)_{+}^{3}-4\left(\frac{1}{2}-q\right)_{+}^{3}
$$

with $H / h=2$ and using the notation $(\cdot)_{+}=\max (\cdot, 0)$. As for the 4th-order, it is given by

$$
f(q)=(1-q)_{+}^{4}-5\left(\frac{3}{5}-q\right)_{+}^{4}+10\left(\frac{1}{5}-q\right)_{+}^{4}
$$

with $H / h=5 / 2$. Finally, the 5 th-order is defined as

$$
f(q)=(1-q)_{+}^{5}-6\left(\frac{2}{3}-q\right)_{+}^{5}+15\left(\frac{1}{3}-q\right)_{+}^{5}
$$

with $H / h=3$.

Contrary to the Gaussian, the Fourier transforms and $F_{2}$ functions of the Wendland and B-Spline kernels depend on the space dimension, and are given in Appendix 3. Figure 1 shows the functions $\widehat{w}\left(K^{*}\right), F_{1}\left(K^{*}\right)$ and $F_{2}\left(K^{*}\right)$ for all the kernels presented here. It confirms that the scaling based on $\sigma$ gives the Fourier transforms a universal shape at small wavenumbers, as said earlier. As a consequence, this is also true for $F_{1}$ and $F_{2}$ (see Appendix 2).

The stability condition (33) gives a stability domain in the $\left(C, C_{\nu}\right)$-plane. Given the complexity of the functions $F_{1}$ and $F_{2}$, the minimum in (33) cannot be algebraically computed, but numerical minima can be obtained from tabulation. Figure 2 depicts the theoretical stability domains resulting from the above-mentioned

\footnotetext{
${ }^{1}$ Strictly speaking, the present 5 th order kernel is only valid for $n=2$ and 3 . The Wendland polynomials depend on the integer part of $n / 2$ (Wendland, 1995). The twice continuously differentiable Wendland kernel for $n=1$ is of 4 th order. Thus, the kernel (39) should be referred to as a 'pseudo-Wendland' in dimension $n=1$.
} 

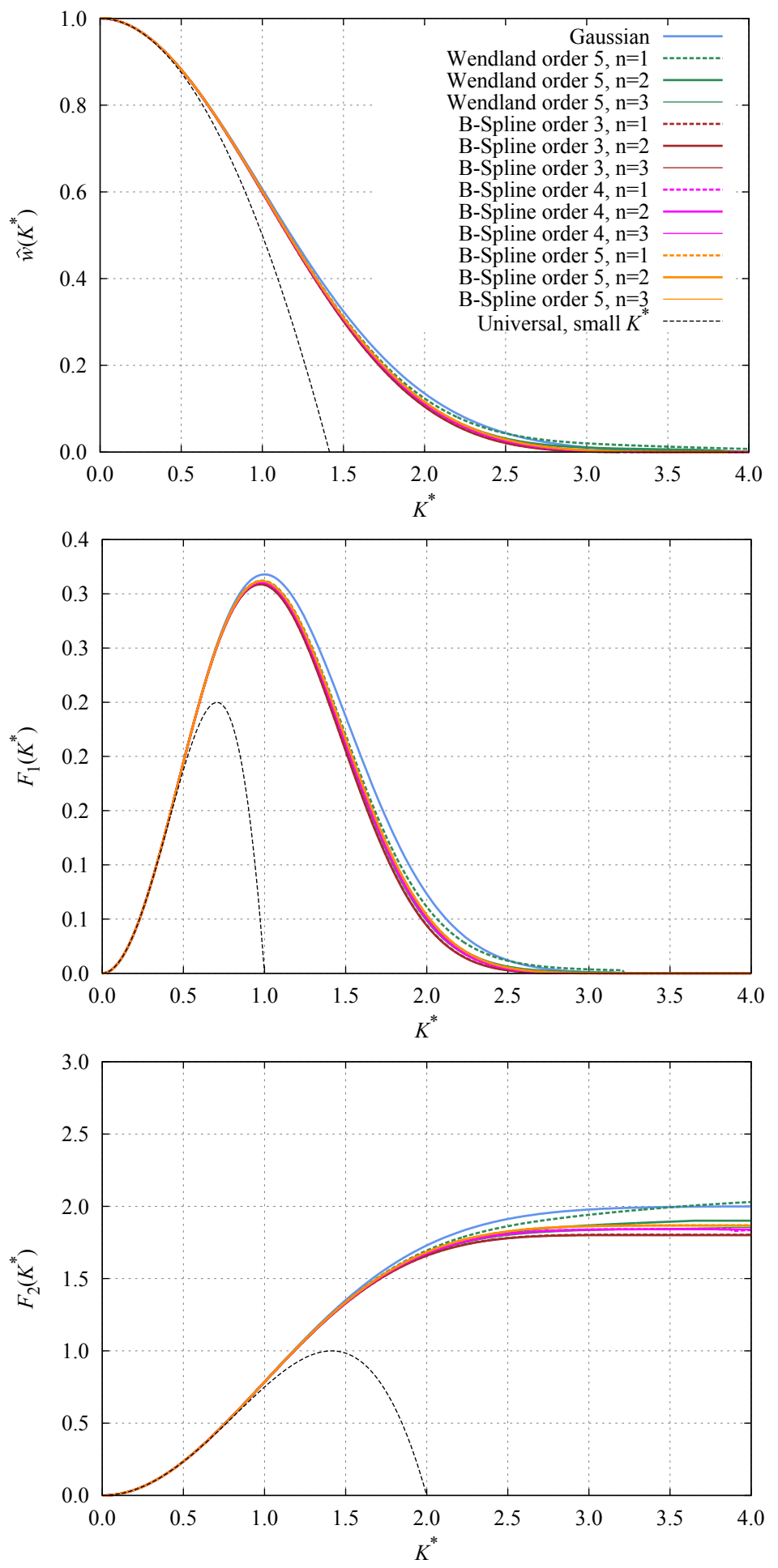

Figure 1: Functions $\widehat{w}\left(K^{*}\right), F_{1}\left(K^{*}\right)$ (defined by eqn $(27)$ ) and $F_{2}\left(K^{*}\right)$ (eqn (18) or (34)) for the various kernels presented here and all space dimensions $n$. The black dotted lines display the universal laws at small wavenumbers (see Appendix 2). 


\begin{tabular}{lcc}
\hline \hline Kernel (dimension $\mathbf{n})$ & $\alpha_{n}$ & $(\sigma / h)^{2}$ \\
\hline \hline Gaussian (all $n)$ & $\frac{1}{(2 \pi)^{n / 2}}$ & 1 \\
\hline \hline Wendland 5th order $(n=1)$ & $\frac{3}{2}$ & $\frac{2}{7}$ \\
\hline Wendland 5th order $(n=2)$ & $\frac{7}{\pi}$ & $\frac{5}{18}$ \\
\hline Wendland 5th order $(n=3)$ & $\frac{21}{2 \pi}$ & $\frac{4}{15}$ \\
\hline \hline B-Spline 3rd order $(n=1)$ & $\frac{8}{3}$ & $\frac{1}{3}$ \\
\hline B-Spline 3rd order $(n=2)$ & $\frac{40}{7 \pi}$ & $\frac{31}{98}$ \\
\hline B-Spline 3rd order $(n=3)$ & $\frac{16}{\pi}$ & $\frac{3}{10}$ \\
\hline \hline B-Spline 4th order $(n=1)$ & $\frac{5^{5}}{768}$ & $\frac{5}{12}$ \\
\hline B-Spline 4th order $(n=2)$ & $\frac{5^{6} 3}{2398 \pi}$ & $\frac{9759}{24416}$ \\
\hline B-Spline 4th order $(n=3)$ & $\frac{5^{6}}{512 \pi}$ & $\frac{23}{60}$ \\
\hline \hline B-Spline 5th order $(n=1)$ & $\frac{3^{5}}{40}$ & $\frac{1}{2}$ \\
\hline B-Spline 5th order $(n=2)$ & $\frac{3^{7} 7}{478 \pi}$ & $\frac{2771}{5736}$ \\
\hline B-Spline 5th order $(n=3)$ & $\frac{3^{7}}{40 \pi}$ & $\frac{7}{15}$ \\
\hline \hline
\end{tabular}

Table 1: Normalizing constants and ratios $\sigma / h$ for various kernels and space dimension $n$.

kernels in all dimensions. It can be seen that in the limit of small numerical Reynolds number $\operatorname{Re}_{0}$ (i.e. small $C / C_{\nu}$ ratio, see (28)), $C_{\nu}$ reaches a plateau. According to (32), the latter is given by $A_{2}=2$ (so that $A_{1}=0$ ), or

$$
C_{\nu, \max }=\frac{2}{\max _{K^{*}} F_{2}\left(K^{*}\right)}
$$

On the other hand, for large $R e_{0}$ (large $C / C_{\nu}$ ratio), the CFL number $C$ reaches a maximum value given by $A_{1}=4$ (so that $A_{2}=0$ ), or

$$
C_{\max }=\frac{2}{\sqrt{\max _{K^{*}} F_{1}\left(K^{*}\right)}}
$$

Generally speaking, $C_{\max }$ cannot be calculated analytically. For the Gaussian kernel, however, $C_{\max }=2 \sqrt{e} \approx 3.30$. The values for the other kernels are computed numerically from (44) and given in Table 2 . On the other hand, $F_{2}$ being an increasing function of $K^{*}$ for all the presently studied kernels, the theoretical values of $C_{\nu \text {, max }}$ can easily be calculated as

$$
C_{\nu, \max }=\frac{2}{\lim _{K^{*} \longrightarrow+\infty} F_{2}\left(K^{*}\right)}
$$




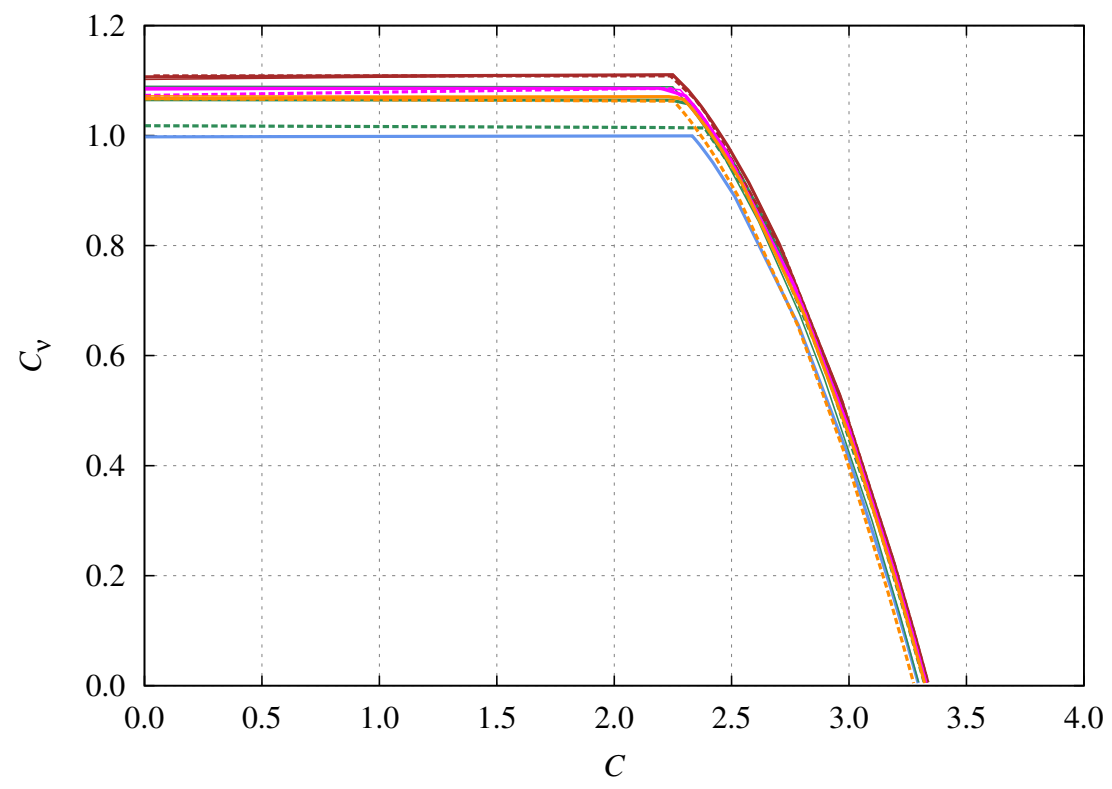

Figure 2: Theoretical stability domains in the $\left(C, C_{\nu}\right)$-plane for the various kernels presented here and all dimensions $n$, according to equation (33). Same colour nomenclature as in Fig. 1.

The theoretical values of $C_{\nu, \max }$ are given by Table 2 . It can be seen, from Figure 2 as well as in Table 2, that the kernel choice and space dimension have only a little effect on the results. This is a result from the fact that we used $\sigma$ as a scaling parameter. In other words, the stability domain is almost universal, especially at large Reynolds numbers (at least for the present choice of time integration scheme and viscous forces, as we will see later). A deeper analysis shows that for small $R e_{0}$, when $C_{\nu}$ exceeds $C_{\nu, \max }$ the instabilities are determined by (theoretically) infinite wavenumbers, while at large $R e_{0}$ finite wavenumbers are unstable when $C$ exceeds $C_{\text {max }}$.

The theoretical stability domains can be compared with the traditional (empirical) SPH stability conditions under viscous forces (see e.g. Morris et al., 1997), which read

$$
\begin{aligned}
& C^{h} \leqslant 0.4 \\
& C_{\nu}^{h} \leqslant 0.125
\end{aligned}
$$

where $C^{h}$ and $C_{\nu}^{h}$ are defined like $C$ and $C_{\nu}$ (eqn (28)) but using $h$ in place of $\sigma$. This choice implies that the stability domains in $\left(C^{h}, C_{\nu}^{h}\right)$-plane are now kernel dependent. Figure 3 displays the stability domains of all kernels with this convention, compared 


\begin{tabular}{lcc}
\hline \hline Kernel (dimension $\mathbf{n})$ & $\mathbf{C}_{\max }$ & $\mathbf{C}_{\nu, \max }$ \\
\hline \hline Gaussian $($ all $n)$ & $2 \sqrt{e} \approx 3.30$ & 1 \\
\hline \hline Wendland 5th order $(n=1)$ & 3.31 & $\frac{14}{15} \approx 0.933$ \\
\hline Wendland 5th order $(n=2)$ & 3.32 & $\frac{36}{35} \approx 1.029$ \\
\hline Wendland 5th order $(n=3)$ & 3.29 & $\frac{15}{14} \approx 1.071$ \\
\hline \hline B-Spline 3rd order $(n=1)$ & 3.34 & $\frac{3}{4 \ln 2} \approx 1.082$ \\
\hline B-Spline 3rd order $(n=2)$ & 3.34 & $\frac{343}{310}=1.106$ \\
\hline B-Spline 3rd order $(n=3)$ & 3.34 & $\frac{10}{9} \approx 1.111$ \\
\hline \hline B-Spline 4th order $(n=1)$ & 3.33 & $\frac{288}{25(25 \ln 5-27 \ln 3)} \approx 1.090$ \\
\hline B-Spline 4th order $(n=2)$ & 3.34 & $\frac{3659348}{3366855} \approx 1.087$ \\
\hline B-Spline 4th order $(n=3)$ & 3.33 & $\frac{25}{23} \approx 1.087$ \\
\hline \hline B-Spline 5th order $(n=1)$ & 3.28 & $\frac{8}{27 \ln 3-32 \ln 2} \approx 1.069$ \\
\hline B-Spline 5th order $(n=2)$ & 3.32 & $\frac{228484}{213} \approx 1.071$ \\
\hline B-Spline 5th order $(n=3)$ & 3.32 & $\frac{15}{14} \approx 1.071$ \\
\hline \hline
\end{tabular}

Table 2: Maximum CFL number $C_{\max }$ and viscous time step $C_{\nu, \max }$ according to the equations (43) and (45) for various kernels and space dimension $n . C_{\nu, \max }$ is given for Morris et al.' s viscous term and without background pressure.

to (46). For all kernels, the stability domain contains the traditional one as given by (46) with a large security margin. Thus, the traditional choice for maximum time step in SPH may be modified, leading to higher time steps. We will come back to this question in Section 3.3.

In order to check the efficiency of the proposed theory, extensive simulations were performed in dimension $n=2$ with all the considered kernels (for the Gaussian kernel, a truncated form was used, as proposed by Cherfils, 2011). The discrete gradient and divergence operators are defined as in (8), thus satisfying first-order consistency of the velocity divergence and momentum conservation. In order to simulate an infinite domain, we use periodic boundary conditions in both directions. We thus consider a square of $40 \times 40$ particles initially at rest (the Galilean invariance of the SPH equations allows this choice) on a Cartesian lattice, and an initial density perturbation of $1 \%$ is set in the right half of the domain (thus, with periodicity it consists of vertical stripes of constant density). Tests were also performed with an initial random noise in density, the results being modified by less than $2 \%$ as regards 
the critical time steps. This test will be widely used in the following, and referred to as the 'infinite flow' case. The computations are run over about 10,000 iterations for several values of $C$, and the flow is considered stable if the space density variations keep bounded by a few percent of the initial mean density. The viscosity is then increased in order to modify $C_{\nu}$ until an instability occurs (generally much before the maximum iteration number, leading to a blowing up). For each value of $C$, it is observed that the computations remain stable until the corresponding critical $C_{\nu}$ is reached, then becomes unstable for immediately higher values. The critical values of $C_{\nu}$ were computed here up to 3 digits. The stability domains obtained by this method are plotted using circles on Figure 3, together with the theoretical domains represented by lines. We used here the $\left(C^{h}, C_{\nu}^{h}\right)$-plane instead of $\left(C, C_{\nu}\right)$ in order to make the comparison between theory and numerical results clearer. The values of $R e_{0}=C / C_{\nu}=C^{h} / C_{\nu}^{h}$ (see eqn (28)) are also given. One can see that the agreement between the theory and the computations is excellent, despite the approximations made (continuous framework, linearisation of the governing equations). Although small discrepancies exist, the shape and size of the stability domains are very well predicted by the present theory, as well as the influence of the kernel (although the latter is negligible when plotted in the $\left(C, C_{\nu}\right)$-plane). One of the main drawbacks of the present theory is that the range of possible wavenumbers is in principle infinite, while in practise $K^{*}$ is upper bounded by $\pi \sigma / \delta r$, since no wave can develop with a wavelength smaller than twice the particle spacing $\delta r$. For the present case, the ratio $h / \delta r$ was set to 1.5 . For this value, at large $K^{*}$ the functions $F_{2}$ have almost reached their asymptotic values, which explains the good agreement between theory and numerical results as regards $C_{\nu, \max }$. This is also why the simulations match the theory with the Gaussian kernel despite the fact that we used a truncated Gaussian, with negative Fourier transform values at large wavenumbers.

Our choice of 1.5 for the ratio $h / \delta r$ may be criticized, since several authors suggest smaller values. On the other hand, our theoretical developments assumed a continuous approximation of the SPH operators, which is more applicable for large $h / \delta r$. Thus we reconducted the present tests with $h / \delta r=1.2$, as recommended by Price (2012). It was found that our numerical critical values of $C_{\nu}$ were only reduced by a very small amount (no more than $2 \%$ ). This shows that the present approach remains valid for moderate densities of particles. Moreover, as pointed out by Morris (1996), Colagrossi et al. (2012) and Dehnen and Aly (2012), the Cartesian particle 
lattice used here is not the closest packing, and hence not a minimum for the internal energy. We reconducted our numerical experiments using an initial triangular particle network, our results being unchanged. This confirms that the continuous approach used in the present theory is correct.

It should be underlined that in the present simulation, it was not possible to keep the simulation stable when the numerical Reynolds number $R e_{0}$, as defined by (29), exceeds a value around 100, slightly depending on the kernel choice. This phenomenon, which is not predicted by the present theory, may result from the discrete properties of SPH. However, the present continuous theory can propose a possible explanation: as $R e_{0}$ increases, the CFL number comes closer to its maximum value $C_{\max }$, for which $A_{1}=4$ and $A_{2}=0$, as stated above. Under such circumstances the polynomial $(30)$ reads $(\chi+1)^{2}=0$, thus $|\chi|=1$. We can conclude that the numerical wave amplitude is not damped but remains constant, thus finite. As a consequence, non-linear terms can be non negligible and enhance instability.

In WCSPH, the numerical speed of sound $c_{0}$ is generally set to 10 times the maximum fluid velocity (Monaghan, 1992). On the other hand, the smoothing length $h$, and thus the space discretization size $\sigma$, is typically 10 to 100 times smaller than the flow size. Thus, if $\nu$ represents the physical (molecular) viscosity then $R e_{0}$ is typically 1 to 10 times smaller than the physical Reynolds number $R e \doteqdot U L / \nu$ where $U$ and $L$ are velocity and length scales, respectively. This means $R e$ cannot excess a maximum value of 100 to 1000 with the present SPH model. In particular, zero-viscosity simulations cannot be performed with the present scheme (a different conclusion will result from the use of the leapfrog scheme, as explained later).

It should be noted that Adami et al. (2013) proposed a transport-velocity form of momentum-conserving SPH which allows to reach larger Reynolds numbers. The authors give no information about the effect of their method on the critical time step. However, it is likely that this newly proposed form of SPH should have benefits in terms of stability, and would deserve a stability analysis based on the present approach.

\section{Study of model variations}

\subsection{Effect of the discrete SPH operators}

We are now going to examine a few variations of the SPH options for discretizing the governing equations, and look at their effects on the stability domain. We begin by looking at the density computation. In many SPH publications (see e.g. Bonet and 
Lok, 1999), an explicit density interpolation is preferred to the continuity equation:

$$
\rho_{a}=\sum_{b} m_{b} w_{a b}
$$

where $w_{a b} \doteqdot w\left(\left|\mathbf{r}_{a}-\mathbf{r}_{b}\right|\right)$. It should be noted that the latter approach defines the density from the particle positions while the continuity equation also involves velocities. In the framework of a continuous time, differentiating (47) gives back the second line of (11), but since the time is discretized, the two approaches are actually different. As a consequence, errors in integrating the continuity equation accumulate and generally require mass diffusion or density smoothing (see e.g. Ferrari et al., 2009). On the other hand, (47) can be used as it is without accumulating density errors.

The continuous form of (47) can be written as

$$
\rho(\mathbf{r})=\int_{\Omega} \rho\left(\mathbf{r}^{\prime}\right) w\left(\left|\mathbf{r}-\mathbf{r}^{\prime}\right|\right) d \mathbf{r}^{\prime}
$$

One can write, using (14) and (16):

$$
\begin{aligned}
\delta \rho(\mathbf{r}) & =\rho_{0} \int_{\Omega} \nabla_{\mathbf{r}} w\left(\left|\mathbf{r}-\mathbf{r}^{\prime}\right|\right) \cdot\left(\delta \mathbf{r}-\delta \mathbf{r}^{\prime}\right) d \mathbf{r}^{\prime} \\
& =i \rho_{0} \exp (-i \mathbf{K} \cdot \mathbf{r}) \widehat{w}\left(K^{*}\right) \mathbf{K}^{*} \cdot \mathbf{R}(t)
\end{aligned}
$$

and then, with (24):

$$
R_{0}=i \widehat{w}\left(K^{*}\right) \mathbf{K}^{*} \cdot \mathbf{R}_{0}
$$

Substituting the latter relation into the first two equations of the system (25) gives (26) again. Thus the stability condition (33) is unchanged, and so is the stability domain. This is illustrated by Figure 4, where the effect of eqn (47) is compared to the original continuity equation on the infinite flow case already presented in Section 2.4. As it can be seen, the computational points match exactly with both approaches for the density estimation.

We now come back to the continuity equation approach, and observe that in the SPH literature, other formulations than (6) are sometimes used for the gradient and divergence operators, given by

$$
\begin{aligned}
& \mathbf{G}^{k}\{A\} \doteqdot \rho^{k} \mathbf{G}\left\{\frac{A}{\rho^{k}}\right\}+\frac{A}{\rho^{k}} \mathbf{G}\left\{\rho^{k}\right\} \\
& D^{k}(\mathbf{A}) \doteqdot \frac{1}{\rho^{k}} D\left\{\rho^{k} \mathbf{A}\right\}-\frac{\mathbf{A}}{\rho^{k}} \cdot \mathbf{G}\left\{\rho^{k}\right\}
\end{aligned}
$$

where $k$ is an arbitrary real number (the superscripts $k$ in the operator notations $\mathbf{G}^{k}$ and $D^{k}$ should not be confused with the exponents in the right-hand-sides of the 
latter definitions). The particular choice $k=1$ comes down to (6), i.e. $D^{1}=D^{-}$ and $\mathbf{G}^{1}=\mathbf{G}^{+}$. Using (4) and (5), the continuous operators are now written

$$
\begin{aligned}
& \mathbf{G}^{k}\{A\}(\mathbf{r})=\int_{\Omega} \frac{\rho\left(\mathbf{r}^{\prime}\right)^{2 k} A(\mathbf{r})+\rho(\mathbf{r})^{2 k} A\left(\mathbf{r}^{\prime}\right)}{\left[\rho(\mathbf{r}) \rho\left(\mathbf{r}^{\prime}\right)\right]^{k}} \nabla_{\mathbf{r}} w\left(\left|\mathbf{r}-\mathbf{r}^{\prime}\right|\right) d \mathbf{r}^{\prime} \\
& D^{k}\{\mathbf{A}\}(\mathbf{r})=-\frac{1}{\rho(\mathbf{r})^{2 k}} \int_{\Omega}\left[\rho(\mathbf{r}) \rho\left(\mathbf{r}^{\prime}\right)\right]^{k}\left[\mathbf{A}(\mathbf{r})-\mathbf{A}\left(\mathbf{r}^{\prime}\right)\right] \cdot \nabla_{\mathbf{r}} w\left(\left|\mathbf{r}-\mathbf{r}^{\prime}\right|\right) d \mathbf{r}^{\prime}
\end{aligned}
$$

in place of (7). Note that the relations (51) are exact when applied to the standard continuous differential operators gradient and divergence (i.e. $\nabla$ in place of $\mathbf{G}$ and $\nabla$. in place of $D$, respectively). Thus, $\mathbf{G}^{k}$ and $D^{k}$ are relevant approximations of the gradient and divergence operators. SPH discrete differential operators can be written from (52):

$$
\begin{aligned}
& \mathbf{G}_{a}^{k}\left\{A_{b}\right\} \doteqdot \sum_{b} V_{b} \frac{\rho_{b}^{2 k} A_{a}+\rho_{a}^{2 k} A_{b}}{\left(\rho_{a} \rho_{b}\right)^{k}} \nabla w_{a b} \\
& D_{a}^{k}\left\{\mathbf{A}_{b}\right\} \doteqdot-\frac{1}{\rho_{a}^{2 k}} \sum_{b} V_{b}\left(\rho_{a} \rho_{b}\right)^{k} \mathbf{A}_{a b} \cdot \nabla w_{a b}
\end{aligned}
$$

The operators (53) are frequently used in SPH, in particular for $k=1$ (as above) and $k=0$ (see e.g. Monaghan, 2005). The operators $\mathbf{G}_{a}^{k}$ and $D_{a}^{k}$ can be proved to be skew-adjoint, leading to interesting numerical properties when used in the momentum and continuity equations, in particular energy conservation (Oger et al., 2007; Violeau, 2012; Mayrhofer et al., 2013).

It is a matter of easy algebra to check that with these new operators the system (20) remains unchanged, regardless of the value of $k$ (see Appendix 1). The conclusion is that the stability domain is unchanged by changing the gradient and divergence operators using (53), for all values of $k$. This prediction was confirmed by numerical results in the case of the infinite flow with $k=0$. Note that the use of the present operators could not allow increasing the maximum Reynolds number mentioned at the end of Section 2.4.

Another possibility consists in using a 'minus' sign to build the gradient operator, leading to

$$
\mathbf{G}_{a}^{-}\left\{A_{b}\right\} \doteqdot-\sum_{b} V_{b}\left(A_{a}-A_{b}\right) \nabla w_{a b}
$$

(we only give here the discrete form). This gradient is zero-order consistent and thus provides more accurate estimations. However, it has been criticized by several authors (see e.g. Monaghan, 1992; Price, 2012) since it does not result from a variational principle, and thus is not momentum-conserving. When applied to (54), 
the present stability analysis shows that the stability domain is unchanged, which is again confirmed by numerical experiments.

Another advantage of the $\mathbf{G}_{a}^{k}$ gradient operators (over $\mathbf{G}_{a}^{-}$) is that they allow using a background pressure, i.e. defining the pressure as

$$
p(\mathbf{r})=\frac{\rho_{0} c_{0}^{2}}{\gamma}\left(\frac{\rho(\mathbf{r})^{\gamma}}{\rho_{0}^{\gamma}}-1+D\right)
$$

instead of (12), where the coefficient $D$ is chosen by the user, but assumed to be positive. This approach is known to avoid artificial cavities near walls. The pressure when $\rho=\rho_{0}$ is now non-zero:

$$
p_{0} \doteqdot \frac{D \rho_{0} c_{0}^{2}}{\gamma}
$$

It is rather easy to show that the linearisation of the momentum equation now gives

$$
\begin{aligned}
& \delta \dot{\mathbf{u}}(\mathbf{r}) \exp (i \mathbf{K} \cdot \mathbf{r}) \\
= & \frac{c_{0}^{2}}{\sigma} \widehat{w}\left(K^{*}\right)\left[-p^{+}\left(\mathbf{K}^{*} \otimes \mathbf{K}^{*}\right) \mathbf{R}(t)+i\left(1-p^{+}\right) R(t) \mathbf{K}^{*}\right] \\
& -\frac{\nu c_{0}}{\sigma^{2}} F_{2}\left(K^{*}\right) \mathbf{U}(t)
\end{aligned}
$$

instead of (17), where we have defined a dimensionless background pressure:

$$
p^{+} \doteqdot \frac{2 D}{\gamma}=\frac{2 p_{0}}{\rho_{0} c_{0}^{2}}
$$

and used the following property of the Fourier transform:

$$
\sigma^{2} \widehat{\nabla_{\tilde{\mathbf{r}}} \nabla_{\tilde{\mathbf{r}}} w}(\mathbf{K})=-\widehat{w}\left(K^{*}\right) \mathbf{K}^{*} \otimes \mathbf{K}^{*}
$$

The first equation of the system (25) being modified accordingly, the equation (26) remains valid if we define differently the function $F_{1}$ :

$$
F_{1}\left(K^{*}, p^{+}\right) \doteqdot K^{* 2} \widehat{w}\left(K^{*}\right)\left[p^{+}+\left(1-p^{+}\right) \widehat{w}\left(K^{*}\right)\right]
$$

in place of (27). The latter equation was already obtained by Dehnen and Aly (2012, their equation (28)). Note that it is now a function of the background pressure as well as the wavenumber, and that $F_{1}\left(K^{*}, 0\right)=F_{1}\left(K^{*}\right)$ as expected. Note also that the last equality of (34) is no longer valid for non-zero background pressure. As for the positivity of $F_{1}$, it remains true if $0 \leqslant \widehat{w}\left(K^{*}\right) \leqslant 1$. We observe that $\widehat{w}(0)=1$, due to the kernel normalising condition. Thus, (60) shows that $F_{1}$ is positive if $\widehat{w}$ is a decreasing and positive function. The latter two properties are true for the Gaussian kernel, but the Wendland kernels Fourier transforms may not be decreasing. On the 
other hand, they satisfy $0 \leqslant \widehat{w}\left(K^{*}\right) \leqslant 1$. As for the splines, they do not satisfy $0 \leqslant \widehat{w}\left(K^{*}\right)$ at large wavenumbers. All this is discussed by Dehnen and Aly (2012).

The stability condition (33) is still valid, using $F_{1}\left(K^{*}, p^{+}\right)$in place of $F_{1}\left(K^{*}\right)$. Figure 5 shows the theoretical effect of the background pressure $p^{+}$in the case of the Wendland kernel of order 5 and $k=1$. The particular case $D=1$ (i.e. $p^{+}=2 / \gamma$ ) was given particular interest as corresponding to compressible gas dynamics. It can be seen that the stability domain is reduced when increasing $p^{+}$, the maximum value of the viscous time step $C_{\nu \text {,max }}$ being unchanged while $C_{\max }$ is reduced, in agreement with (43) and (44), which now reads

$$
C_{\max }=\frac{2}{\sqrt{\max _{K^{*}} F_{1}\left(K^{*}, p^{+}\right)}}
$$

The same figure shows that the numerical results confirm the theoretical predictions for varying background pressure in the case of the infinite flow case. Note that a too large background pressure would require reducing the time step under the traditional criteria given by (46), although this is not yet the case for the maximum value of $p^{+}$presented here.

The viscous operator (9) and (10) due to Morris et al. (1997) may also be changed according to Monaghan and Gingold (1983), where the diffusion coefficient $B$ is assumed to be a constant:

$$
\begin{aligned}
\mathbf{L}^{M G}\{B, \mathbf{A}\}(\mathbf{r}) & \\
& \doteqdot 2(n+2) B \int_{\Omega}\left[\mathbf{A}(\mathbf{r})-\mathbf{A}\left(\mathbf{r}^{\prime}\right)\right] \cdot \frac{\mathbf{r}-\mathbf{r}^{\prime}}{\left|\mathbf{r}-\mathbf{r}^{\prime}\right|^{2}} \nabla_{\mathbf{r}} w\left(\left|\mathbf{r}-\mathbf{r}^{\prime}\right|\right) d \mathbf{r}^{\prime} \\
\mathbf{L}_{a}^{M G}\left\{B, \mathbf{A}_{b}\right\} & \doteqdot 2(n+2) B \sum_{b} V_{b} \mathbf{A}_{a b} \cdot \frac{\mathbf{r}_{a b}}{r_{a b}^{2}} \nabla w_{a b}
\end{aligned}
$$

where $n$ is still the space dimension (see also Colagrossi et al., 2010, or Violeau and Issa, 2006 for non-constant $B$ ). The stability analysis here modifies the viscous term, which in fine appears in the form of $(25)$ but changing the function $F_{2}\left(K^{*}\right)$ with $(n+2) \mathbf{F}_{2}\left(K^{*}\right)$, where $\mathbf{F}_{2}\left(K^{*}\right)$ is a second-order tensor:

$$
\mathbf{F}_{2}\left(K^{*}\right) \doteqdot 2 \sigma^{2} \int_{\Omega}[\exp (-i \mathbf{K} \cdot \tilde{\mathbf{r}})-1] \frac{\tilde{\mathbf{r}}}{\tilde{r}^{2}} \otimes \nabla_{\tilde{\mathbf{r}}} w(\tilde{r}) d \tilde{\mathbf{r}}
$$

Obviously, its trace is equal to $F_{2}\left(K^{*}\right)$ as given by (18). It is proved in Appendix 2 that

$$
\mathbf{F}_{2}\left(K^{*}\right)=\frac{1}{n}\left[F_{2}\left(K^{*}\right)-b\left(K^{*}\right)\right] \mathbf{I}_{n}+\frac{b\left(K^{*}\right)}{K^{* 2}} \mathbf{K}^{*} \otimes \mathbf{K}^{*}
$$


where $b\left(K^{*}\right)$ is found to be the $(n+1)$-moment of the kernel Fourier transform:

$$
b\left(K^{*}\right)=\frac{2}{K^{* n}} \int_{0}^{K^{*}} \kappa^{n+1} \widehat{w}(\kappa) d \kappa
$$

Substituting this result into (25) gives the following equation:

$$
\begin{aligned}
& (\chi-1)\left(\chi-1+\frac{n+2}{n} C_{\nu}\left[F_{2}\left(K^{*}\right)-b\left(K^{*}\right)\right]\right) K^{* 2} \mathbf{U}_{0} \\
= & -\left[\chi C^{2} F_{1}\left(K^{*}\right)+(\chi-1)(n+2) C_{\nu} b\left(K^{*}\right)\right]\left(\mathbf{K}^{*} \otimes \mathbf{K}^{*}\right) \mathbf{U}_{0}
\end{aligned}
$$

in place of (26) which was obtained with Morris et al.'s viscous forces. From that, we obtain a characteristic polynomial identical to (30) but with a modified function for viscous forces:

$$
F_{2}^{M G}\left(K^{*}\right) \doteqdot \frac{n+2}{n}\left[F_{2}\left(K^{*}\right)+(n-1) b\left(K^{*}\right)\right]
$$

which should be used in place of $F_{2}\left(K^{*}\right)$. The stability condition (33) is thus the same, mutatis mutandis. Since the function $F_{1}\left(K^{*}\right)$ is unchanged in comparison to the previous computations, the high Reynolds number limit $C_{\max }$ remains unchanged. On the other hand, the stability domain should now be dimension-dependant for small values of $R e_{0}$, even with the Gaussian kernel. In dimension $n=1, b\left(K^{*}\right)$ is useless and (67) gives $F_{2}^{M G}\left(K^{*}\right)=3 F_{2}\left(K^{*}\right)$ regardless of the kernel choice. In dimensions 2 and 3, the functions $F_{2}^{M G}$ for the Gaussian kernel are given in Appendix 2.

The values of $C_{\nu, \max }$ are modified according to eqn (43), again using $F_{2}^{M G}\left(K^{*}\right)$ in place of $F_{2}\left(K^{*}\right)$. However, contrary to $F_{2}\left(K^{*}\right)$ the $F_{2}^{M G}(K *)$ functions are not monotonically increasing (except for $n=1$ ), but rather reach a maximum for a finite wavenumber before decreasing to their asymptotic value at large wavenumbers. Thus the formula (45) is no longer valid. For the Gaussian kernel, using the above results we find numerically $C_{\nu, \max }=1 / 3 \approx 0.333,0.410$ and 0.458 for $n=1,2$ and 3 , respectively. Note that the stability domain is now smaller for low Reynolds numbers than with the viscous term proposed by Morris et al. (but still larger than Morris et al.'s empirical stability domain (46)). Figure 6 illustrates these results and compares the present theory with numerical results in the case of the infinite flow in dimension $n=2$ with the original discrete gradient and divergence operators $\mathbf{G}^{+}$and $D^{-}$(as in Section 2.4).

As a conclusion of the present section and the previous one, the stability condition (33) is valid for a large range of SPH fluid models, i.e. continuity equation of density interpolation, all traditional gradient and divergence operators, all traditional models 
for viscous forces, provided we use (60) as a generalisation of $F_{1}\left(K^{*}\right),(18)$ for $F_{2}\left(K^{*}\right)$ with Morris et al.'s viscous forces and $F_{2}^{M G}\left(K^{*}\right)$ in place of it for Monaghan and Gingold's viscous forces, see eqns (65) and (67). It is now convenient to rewrite (33) as a condition on the time step $\delta t$. From (33) we get a second-order polynomial in $\delta t$, giving

$$
\delta t=\frac{\nu}{c_{0}^{2}} \min _{K^{*}} \frac{F_{2}\left(K^{*}\right)}{F_{1}\left(K^{*}, p^{+}\right)}\left(\sqrt{1+F_{1}\left(K^{*}, p^{+}\right)\left[\frac{2 R e_{0}}{F_{2}\left(K^{*}\right)}\right]^{2}}-1\right)
$$

(recall $R e_{0}$ is defined by (29)). For small $R e_{0}$, this formula gives

$$
\delta t=\frac{2 \sigma^{2}}{\nu} \frac{1}{\max _{K^{*}} F_{2}\left(K^{*}\right)}=C_{\nu, \max } \frac{\sigma^{2}}{\nu}
$$

in agreement with (43), while at large $R e_{0}$ it yields

$$
\delta t=\frac{2 \sigma}{c_{0}} \frac{1}{\max _{K *} \sqrt{F_{1}\left(K^{*}, p^{+}\right)}}=C_{\max } \frac{\sigma}{c_{0}}
$$

according to (44).

\subsection{Effect of the time marching scheme}

We now investigate the effect of the time marching scheme. The scheme (22) has been preferred so far because of its sequential nature. However, many other schemes are possible, including schemes of order two or more. As an example, Monaghan (2005) gives a second-order (leap-frog) scheme, widely used in the SPH literature for its accuracy properties and time reversibility (in the absence of viscous forces). A stability analysis was performed by Randles and Libersky (1996) with secondorder schemes for the dynamics of solids in dimension $n=1$. Before applying our method to the leap-frog scheme, we continue with first-order schemes, for the sake of simplicity. We will now introduce a fully explicit scheme (used e.g. by De Leffe, 2011), in which the velocities in the right-hand sides of (22) (or (23)) are taken at the previous time step, i.e. $\mathbf{U}\left(t^{m}\right)$ is written in place of $\mathbf{U}\left(t^{m+1}\right)$. In the system (25), the factor $\chi$ in the right-hand-sides of the last two equations are thus removed, and the characteristic equation (30) is replaced by

$$
\chi^{2}-\left(2-A_{2}\right) \chi+1+A_{1}-A_{2}=0
$$


with the same definitions (31) for $A_{1}$ and $A_{2}$. The stability condition $|\chi| \leqslant 1$ is now reached if and only if $A_{1} \leqslant A_{2}$ and $2 A_{2}-A_{1} \leqslant 4$, or

$$
\begin{aligned}
C^{2} & \leqslant C_{\nu} \min _{K^{*}} \frac{F_{2}\left(K^{*}\right)}{F_{1}\left(K^{*}\right)} \\
C_{\nu} & \leqslant \min _{K^{*}} \frac{4+C^{2} F_{1}\left(K^{*}\right)}{2 F_{2}\left(K^{*}\right)}
\end{aligned}
$$

For all the kernels considered in the present work, it was observed that the second of these condition is reached for infinite values of the wavenumber, for which $F_{1}$ tends towards zero while $F_{2}$ has an asymptotic non-zero value (see Section 2.4). On the other hand, the first condition is obtained for very small wavenumbers. Moreover, it is proved in Appendix 2 that $F_{2}\left(K^{*}\right) / F_{1}\left(K^{*}\right)$ tends towards 1 for small $K^{*}$. Thus, the latter conditions may be written as

$$
C^{2} \leqslant C_{\nu} \leqslant \frac{2}{\lim _{K^{*} \longrightarrow+\infty} F_{2}\left(K^{*}\right)}
$$

The values of the right-hand-side of (73) can be found in Table 1, giving $C_{\nu, \max }$ from (45). The stability domain in the $\left(C, C_{\nu}\right)$-plane is thus bounded by a straight line parallel to the $C$-axis and by a parabola starting from the origin. The straight line and the parabola intersect for a critical value of $R e_{0}$ :

$$
R e_{c}=\sqrt{\frac{K^{*} \longrightarrow+\infty}{2} F_{2}\left(K^{*}\right)}
$$

$R e_{c}$ is typically order unity, and is equal to 1 with the Gaussian kernel. Note that the stability domain is again almost independent of the kernel choice. Figure 7 shows that the present theoretical result is in excellent agreement with our simulations in the case of the infinite flow case with the B-Spline of order 5 . This shape was already predicted by De Leffe (2011) in his PhD from different considerations. De Leffe used a discrete point of view contrary to the present theory, generalizing to arbitrary dimension $n$ the work done by Swegle et al. (1995), but he provided a validation in one dimension only, and the agreement between his numerical results and his theory is less good than the present study (unfortunately his work was never published in English).

Note that the stability domain is significantly smaller at 'large' $R e_{0}\left(R e_{0}>R e_{c}\right)$ than with the sequential scheme investigated in the previous two sections, although they match at very small $R e_{0}\left(R e_{0}<R e_{c}\right)$. Moreover it was hardly possible to exceed $R e_{0}=10$, and for the largest possible numerical Reynolds number the time 
step takes very small values. This can be explained by rewriting (73) in terms of the time step:

$$
\delta t \leqslant \min \left[\frac{\nu}{c_{0}^{2}} ; \frac{\sigma^{2}}{\nu} \frac{2}{\lim _{K^{*}+\infty} F_{2}\left(K^{*}\right)}\right]
$$

The first of the above conditions is the most important at large $R e_{0}$, giving a very small $\delta t$ because of the factor $c_{0}^{2}$. It is thus recommended to avoid this time integration scheme as regards the optimum choice of the time step. Note that with the present explicit scheme the stability conditions are no longer satisfied with the traditional criteria (46), except for very small Reynolds numbers.

We now come to the second-order leapfrog scheme. Following Monaghan (2005), we write it as

$$
\begin{aligned}
\frac{\mathbf{u}\left(t^{m+1 / 2}\right)-\mathbf{u}\left(t^{m}\right)}{\delta t / 2} & =\mathbf{F}\left(t^{m}\right) \\
\frac{\mathbf{r}\left(t^{m+1}\right)-\mathbf{r}\left(t^{m}\right)}{\delta t} & =\mathbf{u}\left(t^{m+1 / 2}\right) \\
\rho\left(t^{m+1}\right) & =\sum_{b} m_{b} w_{a b}^{m+1} \\
\frac{\mathbf{u}\left(t^{m+1}\right)-\mathbf{u}\left(t^{m+1 / 2}\right)}{\delta t / 2} & =\mathbf{F}\left(t^{m+1}\right)
\end{aligned}
$$

In comparison to the first-order scheme (22), the leapfrog consists of estimating velocities at half-integer times. This is why the density is calculated from (47), avoiding the use of velocities at time $t^{m+1}$. For the same reason, in the force $\mathbf{F}\left(t^{m+1}\right)$ all parameters are considered at time $t^{m+1}$ except the velocity in the viscous term, which is taken at $t^{m}$. It should be emphasized that with this choice, the present scheme is not exactly second-order in time. To achieve second-order, an additional step should be used to estimate 'intermediate' velocities $u^{*}$ at time $t^{m+1}$ from $\mathbf{F}\left(t^{m}\right)$; the $u^{*}$ should then be used in the viscous force on the last line of (76). However, this modification would not affect the stability domain, as evidenced by algebraic computations. It is possible to rearrange (76) using only integer times to give

$$
\begin{aligned}
& \mathbf{r}\left(t^{m+1}\right)=\mathbf{r}\left(t^{m}\right)+\delta t \mathbf{u}\left(t^{m}\right)+\frac{\delta t^{2}}{2} \mathbf{F}\left(t^{m}\right) \\
& \rho\left(t^{m+1}\right)=\sum_{b} m_{b} w_{a b}^{m+1} \\
& \mathbf{u}\left(t^{m+1}\right)=\mathbf{u}\left(t^{m}\right)+\frac{\delta t}{2}\left[\mathbf{F}\left(t^{m}\right)+\mathbf{F}\left(t^{m+1}\right)\right]
\end{aligned}
$$

Applying our method to this scheme leads to the following eigenvalue problem:

$$
\left(\chi-1+A_{2}\right)(\chi-1) K^{* 2} \mathbf{R}_{0}=-\chi \frac{A_{1}}{2}\left[\left(2-\frac{A_{2}}{2}\right) \chi+\frac{A_{2}}{2}\right]\left(\mathbf{K}^{*} \otimes \mathbf{K}^{*}\right) \mathbf{R}_{0}
$$


in place of (26), using the same definitions (31) for $A_{1}$ and $A_{2}$. In case of background pressure, it is easy to show that the result is unchanged provided we use the modified function $F_{1}\left(K^{*}, p^{+}\right)$defined for the first-order schemes (Section 3.1, eqn (60)). Again, we need to study separately the eigenvalues of the matrix $\mathbf{K}^{*} \otimes \mathbf{K}^{*}$. The eigenvalue $K^{* 2}$ gives a second-order polynomial for $\chi$ similar to (30), leading to the stability condition $A_{1} \leqslant 4$, or $C \leqslant C_{\max }$ where $C_{\max }$ is defined by (44). On the other hand, the zero eigenvalue gives $\chi=1-A_{2}$, with the stability condition $A_{2} \leqslant 2$ or $C_{\nu} \leqslant C_{\nu, \max }$ where $C_{\nu, \max }$ is defined by (45). Finally, the theoretical stability domain is a rectangle of size $C_{\max } \times C_{\nu, \max }$ in the $\left(C, C_{\nu}\right)$-plane, giving:

$$
\delta t \leqslant \min \left[\frac{2}{\sqrt{\max _{K^{*}} F_{1}\left(K^{*}, p^{+}\right)}} \frac{\sigma}{c_{0}} ; \frac{4}{\lim _{K^{*} \longrightarrow+\infty} F_{2}\left(K^{*}\right)} \frac{\sigma^{2}}{\nu}\right]
$$

The upper right corner of the rectangle corresponds to $C=C_{\nu}$, i.e. $R e_{0}=1$. As depicted by Figure 8, our numerical tests confirm these results in the infinite flow case, although $C$ should be decreased for large $R e_{0}$. In this case, however, the leapfrog allowed increasing $R e_{0}$ arbitrarily, and even removing the viscous forces without affecting stability. The numerical maximum value of $C$ in this case is a bit less than 2.

As a conclusion of the present section, it should be emphasized that contrary to the space integration operators, which have little effect on the stability properties as illustrated in Section 3.1 (except the model used for viscous forces), the time integration scheme can have a serious influence on the stability domain. The leapfrog scheme is recommended for its ability to model inviscid unbounded flows.

\subsection{Application to real flows}

The previous theoretical results and numerical validations were obtained from the hypothesis of an infinite flow with constant velocity. In order to extend the present theory to real SPH simulations, it is necessary to investigate the effects of solid walls (from now on the space dimension is $n=2$ or $n=3$ ). For this purpose, we first need to take a look at the influence of a velocity gradient in the infinite flow case. Thus, we come back to the considerations of Section 2 (i.e. first-order operators and no background pressure) and consider as a reference state a linear velocity field:

$$
\mathbf{u}(\mathbf{r})=\frac{z}{T} \mathbf{e}_{x}
$$

where $T$ is a time scale (the inverse of the scalar strain rate) and $z=\mathbf{r} \cdot \mathbf{e}_{z}, \mathbf{e}_{x}$ and $\mathbf{e}_{z}$ being orthogonal unit vectors. With this assumption, the linearised system is more 
complex, and the characteristic equation for the amplification factor $\chi$ involves new terms with $C_{T} \doteqdot \delta t / T$ as a new dimensionless parameter. Seeking for the eigenvalues of the matrix would finally lead to a polynomial of order 5 for $\chi$. It is known that such polynomials have no analytical solutions except in special cases, so that this problem cannot be solved by the present method. However, it is not necessary to solve such an equation in general. Indeed, with the stability criterion (33) the typical order of magnitude of $\delta t$ is given by $\sigma / c_{0}$, so that $C_{T}$ is order $\sigma /\left(c_{0} T\right) \ll L /(U T)$, where $L$ and $U$ are some flow length and velocity scales, respectively. Thus, contrary to $C$ and $C_{\nu}, C_{T}$ is in general very small and should be neglected, so that the characteristic equation finally goes back to (26). Moreover, it is easy to check that the model variations of Section 3.1 do not affect this result. In other words, a linear velocity gradient should not modify the stability domain. We certainly can extend this conclusion to arbitrary velocity gradients, which can be locally linearised. This result was numerically checked with an infinite flow driven by a sinusoidal force, leading to a sinusoidal velocity field:

$$
\mathbf{u}(\mathbf{r})=\alpha c_{0} \sin \left(\frac{2 \pi z}{L}\right) \mathbf{e}_{x}
$$

This choice allows using periodicity (with space periodicity $L$ ) in order to simulate an unbounded flow. We selected two values of the ratio $\alpha$, which is a dimensionless measure of the velocity gradient, i.e. $\alpha=0.05$ and $\alpha=0.1$. With the traditional choice for $c_{0}$ in WCSPH (i.e. 10 times the maximum flow velocity), this gives significant values for the velocity gradient (here $T \sim L /\left(2 \pi \alpha c_{0}\right)$ ). It should be noted that it was not possible to keep this flow stable for a long time without using background pressure. Thus $p^{+}$is set to $1 / 7 \approx 0.14$ (i.e. $D=0.5$ in (55)). The kind of instability occurring when using $p^{+}=0$ was not a blowing up, but rather a particle disorder (a well-known property of momentum-conserving SPH with vanishing pressure). This leads to significant fluctuations in density, although it does not affect the velocity field too much. However, in the present numerical stability tests, it was considered that stability is achieved only if the particles keep parallel paths 'indefinitely'. This was only possible here for $R e_{0}$ less than about 50 . Figure 9 shows the stability domain of the sinusoidal flow (81) with the Gaussian kernel. It also includes, for comparison, the infinite flow case without velocity gradient, with and without background pressure. We can see that the velocity gradient does not affect the shape and size of the domain, regardless of $\alpha$.

The influence of walls is more difficult to handle from theoretical considerations. 
The first reason is that there is no unique standard model for modelling solid walls in SPH for fluids. One of the most recent and successful approach is based on wall renormalisation functions (see e.g. Ferrand et al., 2012), which will be used in the following. The other reason is that the numerical fluctuations now cannot be written in the form (14) since they must satisfy the wall boundary conditions. This is achieved by introducing a second numerical wave reflected by the wall. However, even in the case of plane wall the problem remains difficult and will not be solved here. In order to address the question of the stability domain for bounded flows, we will proceed from numerical experiments. We first show the stability domain of a laminar steady Poiseuille flow in a pipe (see e.g. Basa et al., 2009), with the Wendland order 5 kernel and background pressure $p^{+}=1 / 7$ as above. As in the infinite flow case, an initial density perturbation of $1 \%$ was imposed. Note that we now have lost the isotropy of the problem, so that near the walls the problem should depend on the direction of the wavevector $\mathbf{K}^{*}$ (this was already true with the velocity gradient case above). We thus tested two different positions of the initial density discontinuity: horizontal (i.e. parallel to the walls) and vertical. Figure 10 shows that in both cases the domain is very similar to the present theory (where the flow is assumed unbounded) for large values of $R e_{0}$, but small $R e_{0}$ lead to a smaller value of $C_{\nu}$. This result, obtained with the first-ordersequential time integrator, looks not surprising since the walls are liable to generate additional instabilities by increasing the strain. However, with the leapfrog scheme the stability domain is very close to the theory even at small Reynolds numbers with this flow, as depicted by Figure 8. On the other hand, even with the leapfrog scheme it was not possible to exceed $R e_{0} \sim 100$ in the presence of walls. The simulations were run over about 30,000 time steps, the particles keeping their paths parallel to the walls. However, additional tests show that some disturbances appear around 1.5 million iterations. It is already known that wall shear flows with SPH remain problematic in long-term simulations, as pointed out by Basa et al. (2009).

Finally, two 2-D test cases were performed from the usual SPH literature: the laminar lid-driven cavity flow (see e.g. Lee et al., 2008) and the collapse of a water column on a triangular wedge (Ferrand et al., 2012). Figure 11 shows the first one, with a comparison against a Finite Volume method and the reference results by Ghia et al. (1982). The lid of the square (of size $L$ ) moves from left to right at a constant velocity $U$ and drives the fluid through viscous shear stress. The curves 
represent the profiles of dimensionless velocity components along the two centerlines, where the dimensionless velocity is defined as $\mathbf{u}^{+} \doteqdot \mathbf{u} / U$. The results are plotted after convergence for a physical Reynolds number $R e \doteqdot U L / \nu=400$. Figure 12 shows the second test case, which is unsteady: a rectangular column of fluid falls down into a rectangular tank with a wedge in the middle of the bottom, generating high distortion and splash-up. The adimensional time is defined as $t^{+}=t \sqrt{g / L}$, where $L$ is the initial height of the fluid column and $g$ the gravity acceleration (note that gravity should have no influence in the linearisation made in Section 2.2). The physical Reynolds number is $R e \doteqdot\left(g L^{3}\right)^{1 / 2} / \nu=313$.

Figure 13 shows the results obtained for the lid-driven cavity and water collapse cases. The lid-driven cavity needed background pressure to keep stable (we tested $p^{+}=1 / 7$ as above, as well as $p^{+}=2 / 7$ corresponding to compressible gas dynamics). Although still larger than the traditional SPH stability domain, the present 'experimental' stability domains are smaller than for the infinite flow case. The lid-driven cavity stability domain remains close to the Poiseuille flow, while the water collapse case presents significant differences, especially at large $R e_{0}$. This is likely to be due to the fact that this flow is highly unsteady, since instabilities can appear without having the time to develop before the flow has moved to a more stable regime. Generally speaking, with the Wendland kernel used in the present section, the time step can be increased by a factor close to 2 in comparison with the traditional empirical condition (46).

\section{Conclusion}

A theoretical model was built in order to better understand how the time step should be upper bounded in SPH simulations for weakly compressible viscous flows. For unbounded flow and without velocity gradient, using continuous SPH differential operators and von Neumann linearising method, it was proved that the time step $\delta t$ should be chosen so that the dimensionless numbers $C \doteqdot c_{0} \delta t / \sigma$ and $C_{\nu} \doteqdot \nu \delta t / \sigma^{2}$ determine a point living within some stability domain. The latter is determined by two functions of the dimensionless numerical wavenumber $K^{*}$, both depending on the SPH kernel Fourier transform. When using the kernel standard deviation in place of the smoothing length to scale our results, the stability domain is almost universal, irrespective of the kernel choice, for a given time integrationscheme and without background pressure.

Numerical tests confirm that the present theory is correct, for various kernels 
widely used in the SPH literature. The effect of the ratio smoothing length/particle size was tested, as well as the initial particle configuration (Cartesian and triangular packaging), without noticeable effects. However, in our simulations with first-order time integration schemes the numerical Reynolds number $R e_{0} \doteqdot c_{0} \sigma / \nu$ could not exceed a maximum value of about 100 without leading to a blowing up.

Additional developments were carried out in order to study the effect of the density computation and of the SPH operators (gradient, divergence). It was found that these modifications do not affect the stability domain, as confirmed by numerical experiments. On the other hand, the model used to compute the viscous forces has an influence, the model proposed by Morris et al. (1997) allowing a larger time step than the one by Monaghan and Gingold (1983), especially at small $R e_{0}$. The background pressure in the state equation also influences stability, by reducing the stability domain. The effect of the time marching schemes was also investigated, showing that a sequential time scheme leads to a larger stability domain than an explicit scheme. Besides, the leapfrog scheme has a moderate influence on the stability domain, but allows very high values of $R e_{0}$. Again, numerical tests support all these theoretical achievements with an excellent accuracy. Accordingly, a leapfrog scheme with Morris et al.'s viscous forces and a small background pressure should be preferred in practical applications.

Finally, the effects of solid walls and unsteady flows were tested from numerical tests. With a first-order scheme, the stability domain at small $R e_{0}$ is reduced in the case of a steady laminar Poiseuille flow in a pipe, as well as for the lid-driven cavity case and the collapse of a water column on a triangular wedge. On the other hand, with the leapfrog scheme the solid walls do not seem to have any influence on the stability domain. Generally speaking, it appears that the experimental stability domains always include the traditional SPH stability criterion for the time step, i.e. $C^{h} \leqslant 0.4$ and $C_{\nu}^{h} \leqslant 0.125$, with a security margin. Hence, these critical values could be increased in practise. A complete theory of SPH stability for viscous fluids including walls would require further research.

In the existing literature on the SPH method for fluids, there are so many different options that it is impossible to study all of them in a single paper. However, the present theory constitutes a framework for further study on SPH numerical stability properties. In particular, it should not be an issue to investigate deeper the influence of time marching schemes. Similarly, appropriate stability criteria could be found 
for SPH surface tension models (see e.g. Hu and Adams, 2006), Incompressible SPH (ISPH, see Lee et al., 2008) and density smoothing procedures (Ferrari et al., 2009). It should also be possible to apply the present method to solid computations through SPH, see e.g. Campbell et al. (2000).

\section{Appendices}

\subsection{Appendix 1}

We investigate the sensitivity of the system (7) to small variations in the particle positions, densities and velocities. As in the standard SPH method, the masses of all particles will be considered as constant. Denoting by $d m^{\prime}=\rho\left(\mathbf{r}^{\prime}\right) d \mathbf{r}^{\prime}$ the infinitesimal mass contained by the volume element $d \mathbf{r}^{\prime}$, and considering the state equation (12), the system (7) now reads

$$
\begin{aligned}
\dot{\mathbf{u}}(\mathbf{r})= & -\frac{\rho_{0} c_{0}^{2}}{\gamma} \int_{\Omega}\left[\begin{array}{c}
\frac{1}{\rho(\mathbf{r})^{2}}\left(\frac{\rho(\mathbf{r})^{\gamma}}{\rho_{0}^{\gamma}}-1\right) \\
+\frac{1}{\rho\left(\mathbf{r}^{\prime}\right)^{2}}\left(\frac{\rho\left(\mathbf{r}^{\prime}\right)^{\gamma}}{\rho_{0}^{\gamma}}-1\right)
\end{array}\right] \nabla_{\mathbf{r}} w\left(\left|\mathbf{r}-\mathbf{r}^{\prime}\right|\right) d m^{\prime} \\
& +2 \mu \int_{\Omega} \frac{1}{\rho(\mathbf{r}) \rho\left(\mathbf{r}^{\prime}\right)}\left[\mathbf{u}(\mathbf{r})-\mathbf{u}\left(\mathbf{r}^{\prime}\right)\right] \frac{\mathbf{r}-\mathbf{r}^{\prime}}{\left|\mathbf{r}-\mathbf{r}^{\prime}\right|^{2}} \cdot \nabla_{\mathbf{r}} w\left(\left|\mathbf{r}-\mathbf{r}^{\prime}\right|\right) d m^{\prime} \\
\dot{\rho}(\mathbf{r})= & \int_{\Omega}\left[\mathbf{u}(\mathbf{r})-\mathbf{u}\left(\mathbf{r}^{\prime}\right)\right] \cdot \nabla_{\mathbf{r}} w\left(\left|\mathbf{r}-\mathbf{r}^{\prime}\right|\right) d m^{\prime}
\end{aligned}
$$

Suppose the positions, velocities and densities are modified by small arbitrary amounts $\delta \mathbf{r}, \delta \rho(\mathbf{r})$ and $\delta \mathbf{u}(\mathbf{r})$, respectively. Then, keeping $d m^{\prime}$ constant and using the property $\nabla_{\mathbf{r}} w\left(\left|\mathbf{r}-\mathbf{r}^{\prime}\right|\right)=-\nabla_{\mathbf{r}^{\prime}} w\left(\left|\mathbf{r}-\mathbf{r}^{\prime}\right|\right)$, the fluid acceleration is modified by $\delta \dot{\mathbf{u}}(\mathbf{r}):$

$$
\begin{aligned}
\delta \dot{\mathbf{u}}(\mathbf{r})= & -\frac{\rho_{0} c_{0}^{2}}{\gamma} \int_{\Omega}\left[\begin{array}{c}
\frac{1}{\rho(\mathbf{r})^{2}}\left(\frac{\rho(\mathbf{r})^{\gamma}}{\rho_{0}^{\gamma}}-1\right) \\
+\frac{1}{\rho\left(\mathbf{r}^{\prime}\right)^{2}}\left(\frac{\rho\left(\mathbf{r}^{\prime}\right)^{\gamma}}{\rho_{0}^{\gamma}}-1\right)
\end{array}\right] \nabla_{\mathbf{r}} \nabla_{\mathbf{r}} w\left(\left|\mathbf{r}-\mathbf{r}^{\prime}\right|\right)\left(\delta \mathbf{r}-\delta \mathbf{r}^{\prime}\right) d m^{\prime} \\
& -\frac{\rho_{0} c_{0}^{2}}{\gamma} \int_{\Omega} \delta \rho(\mathbf{r})\left[(\gamma-2) \frac{\rho(\mathbf{r})^{\gamma-3}}{\rho_{0}^{\gamma}}+\frac{2}{\rho(\mathbf{r})^{3}}\right] \nabla_{\mathbf{r}} w\left(\left|\mathbf{r}-\mathbf{r}^{\prime}\right|\right) d m^{\prime} \\
& -\frac{\rho_{0} c_{0}^{2}}{\gamma} \int_{\Omega} \delta \rho\left(\mathbf{r}^{\prime}\right)\left[(\gamma-2) \frac{\rho\left(\mathbf{r}^{\prime}\right)^{\gamma-3}}{\rho_{0}^{\gamma}}+\frac{2}{\rho\left(\mathbf{r}^{\prime}\right)^{3}}\right] \nabla_{\mathbf{r}} w\left(\left|\mathbf{r}-\mathbf{r}^{\prime}\right|\right) d m^{\prime} \\
& +2 \mu \int_{\Omega} \frac{1}{\rho(\mathbf{r}) \rho\left(\mathbf{r}^{\prime}\right)}\left[\mathbf{u}(\mathbf{r})-\mathbf{u}\left(\mathbf{r}^{\prime}\right)\right] \nabla_{\mathbf{r}}\left[\frac{\mathbf{r}-\mathbf{r}^{\prime}}{\left|\mathbf{r}-\mathbf{r}^{\prime}\right|^{2}} \cdot \nabla_{\mathbf{r}} w\left(\left|\mathbf{r}-\mathbf{r}^{\prime}\right|\right)\right] \cdot\left(\delta \mathbf{r}-\delta \mathbf{r}^{\prime}\right) d m^{\prime} \\
& -2 \mu \int_{\Omega} \frac{1}{\rho(\mathbf{r}) \rho\left(\mathbf{r}^{\prime}\right)}\left[\frac{\delta \rho(\mathbf{r})}{\rho(\mathbf{r})}+\frac{\delta \rho\left(\mathbf{r}^{\prime}\right)}{\rho\left(\mathbf{r}^{\prime}\right)}\right]\left[\mathbf{u}(\mathbf{r})-\mathbf{u}\left(\mathbf{r}^{\prime}\right)\right] \frac{\mathbf{r}-\mathbf{r}^{\prime}}{\left|\mathbf{r}-\mathbf{r}^{\prime}\right|^{2}} \cdot \nabla_{\mathbf{r}} w\left(\left|\mathbf{r}-\mathbf{r}^{\prime}\right|\right) d m^{\prime} \\
& +2 \mu \int_{\Omega} \frac{1}{\rho(\mathbf{r}) \rho\left(\mathbf{r}^{\prime}\right)}\left[\delta \mathbf{u}(\mathbf{r})-\delta \mathbf{u}\left(\mathbf{r}^{\prime}\right)\right] \frac{\mathbf{r}-\mathbf{r}^{\prime}}{\left|\mathbf{r}-\mathbf{r}^{\prime}\right|^{2}} \cdot \nabla_{\mathbf{r}} w\left(\left|\mathbf{r}-\mathbf{r}^{\prime}\right|\right) d m^{\prime}
\end{aligned}
$$


Similarly, the density time derivative is modified by the following amount:

$$
\begin{aligned}
\delta \dot{\rho}(\mathbf{r})= & \int_{\Omega}\left[\mathbf{u}(\mathbf{r})-\mathbf{u}\left(\mathbf{r}^{\prime}\right)\right] \cdot \nabla_{\mathbf{r}} \nabla_{\mathbf{r}} w\left(\left|\mathbf{r}-\mathbf{r}^{\prime}\right|\right)\left(\delta \mathbf{r}-\delta \mathbf{r}^{\prime}\right) d m^{\prime} \\
& +\int_{\Omega}\left[\delta \mathbf{u}(\mathbf{r})-\delta \mathbf{u}\left(\mathbf{r}^{\prime}\right)\right] \cdot \nabla_{\mathbf{r}} w\left(\left|\mathbf{r}-\mathbf{r}^{\prime}\right|\right) d m^{\prime}
\end{aligned}
$$

We will now consider a numerical fluctuation around a homogeneous state corresponding to the fluid equilibrium, i.e. $\mathbf{u}(\mathbf{r})=\mathbf{c s t}$ and $\rho(\mathbf{r})=\rho_{0}$, giving

$$
\begin{aligned}
\delta \dot{\mathbf{u}}(\mathbf{r})= & -\frac{c_{0}^{2}}{\rho_{0}} \int_{\Omega}\left[\delta \rho(\mathbf{r})+\delta \rho\left(\mathbf{r}^{\prime}\right)\right] \nabla_{\mathbf{r}} w\left(\left|\mathbf{r}-\mathbf{r}^{\prime}\right|\right) d \mathbf{r}^{\prime} \\
& +2 \nu \int_{\Omega}\left[\delta \mathbf{u}(\mathbf{r})-\delta \mathbf{u}\left(\mathbf{r}^{\prime}\right)\right] \frac{\mathbf{r}-\mathbf{r}^{\prime}}{\left|\mathbf{r}-\mathbf{r}^{\prime}\right|^{2}} \cdot \nabla_{\mathbf{r}} w\left(\left|\mathbf{r}-\mathbf{r}^{\prime}\right|\right) d \mathbf{r}^{\prime}
\end{aligned}
$$

with $\nu \doteqdot \mu / \rho_{0}$ the kinematic viscosity, and

$$
\delta \dot{\rho}(\mathbf{r})=\rho_{0} \int_{\Omega}\left[\delta \mathbf{u}(\mathbf{r})-\delta \mathbf{u}\left(\mathbf{r}^{\prime}\right)\right] \cdot \nabla_{\mathbf{r}} w\left(\left|\mathbf{r}-\mathbf{r}^{\prime}\right|\right) d \mathbf{r}^{\prime}
$$

In case of the generalized operators (52), we can check that the system (20) remains unchanged, regardless of the value of $k$. For example, the fluctuation of the density time derivative is now given by

$$
\begin{aligned}
\delta \dot{\rho}(\mathbf{r})= & \delta \int_{\Omega}\left[\frac{\rho\left(\mathbf{r}^{\prime}\right)}{\rho(\mathbf{r})}\right]^{k}\left[\mathbf{u}(\mathbf{r})-\mathbf{u}\left(\mathbf{r}^{\prime}\right)\right] \cdot \nabla_{\mathbf{r}} w\left(\left|\mathbf{r}-\mathbf{r}^{\prime}\right|\right) d m^{\prime} \\
= & \int_{\Omega}\left[\frac{\rho\left(\mathbf{r}^{\prime}\right)}{\rho(\mathbf{r})}\right]^{k}\left[\mathbf{u}(\mathbf{r})-\mathbf{u}\left(\mathbf{r}^{\prime}\right)\right]^{T} \nabla_{\mathbf{r}} \nabla_{\mathbf{r}} w\left(\left|\mathbf{r}-\mathbf{r}^{\prime}\right|\right)\left(\delta \mathbf{r}-\delta \mathbf{r}^{\prime}\right) d m^{\prime} \\
& +\int_{\Omega}\left[\frac{\rho\left(\mathbf{r}^{\prime}\right)}{\rho(\mathbf{r})}\right]^{k}\left[\delta \mathbf{u}(\mathbf{r})-\delta \mathbf{u}\left(\mathbf{r}^{\prime}\right)\right] \cdot \nabla_{\mathbf{r}} w\left(\left|\mathbf{r}-\mathbf{r}^{\prime}\right|\right) d m^{\prime} \\
& +(k-1) \int_{\Omega}\left[\frac{\rho\left(\mathbf{r}^{\prime}\right)}{\rho(\mathbf{r})}\right]^{k-1}\left[\frac{\delta \rho\left(\mathbf{r}^{\prime}\right)}{\rho\left(\mathbf{r}^{\prime}\right)}-\frac{\delta \rho(\mathbf{r})}{\rho(\mathbf{r})}\right]\left[\mathbf{u}\left(\mathbf{r}^{\prime}\right)-\mathbf{u}(\mathbf{r})\right] \cdot \nabla_{\mathbf{r}} w\left(\left|\mathbf{r}-\mathbf{r}^{\prime}\right|\right) d m^{\prime}
\end{aligned}
$$

instead of (84). From an equilibrium state defined by $\mathbf{u}(\mathbf{r})=\mathbf{c s t}$ and $\rho(\mathbf{r})=\rho_{0}$ as previously, this gives (84) again.

\subsection{Appendix 2}

The functions $F_{1}$ and $F_{2}$ introduced in Sections 2.2 and 2.4 (eqns (27) and (18), respectively) are linked to each other in the absence of background pressure. Indeed, differentiating (18) with respect to the dimensionless wavevector $\mathbf{K}^{*}$ gives

$$
\begin{aligned}
F_{2}^{\prime}\left(K^{*}\right) & =\nabla_{\mathbf{K}^{*}} F_{2}\left(K^{*}\right) \cdot \frac{\mathbf{K}^{*}}{K^{*}} \\
& =-2 i \sigma\left[\int_{\Omega} \exp (-i \mathbf{K} \cdot \tilde{\mathbf{r}}) \tilde{\mathbf{r}} \frac{\tilde{\mathbf{r}}}{\tilde{r}^{2}} \cdot \nabla_{\tilde{\mathbf{r}}} w(\tilde{r}) d \tilde{\mathbf{r}}\right] \cdot \frac{\mathbf{K}^{*}}{K^{*}}
\end{aligned}
$$


Now, the vectors $\tilde{\mathbf{r}}$ and $\nabla_{\tilde{\mathbf{r}}} w(\tilde{r})$ are colinear since $w$ is isotropic. Thus we can swap them into the integral. Writing $\tilde{\mathbf{r}} \cdot \tilde{\mathbf{r}}=\tilde{r}^{2}$, we get:

$$
\begin{aligned}
F_{2}^{\prime}\left(K^{*}\right) & =-2 i \sigma\left[\int_{\Omega} \exp (-i \mathbf{K} \cdot \tilde{\mathbf{r}}) \nabla_{\tilde{\mathbf{r}}} w(\tilde{r}) d \tilde{\mathbf{r}}\right] \cdot \frac{\mathbf{K}^{*}}{K^{*}} \\
& =-2 i \sigma \widehat{\nabla_{\tilde{\mathbf{r}}} w}(\mathbf{K}) \cdot \frac{\mathbf{K}^{*}}{K^{*}} \\
& =2 K^{*} \widehat{w}\left(K^{*}\right)
\end{aligned}
$$

where we have used (16). Finally, with the definition (27) we have

$$
F_{2}^{\prime}\left(K^{*}\right)=2 \sqrt{F_{1}\left(K^{*}\right)}
$$

which is (34).

The tensorial function $\mathbf{F}_{2}\left(K^{*}\right)$ defined by (63) obviously satisfy

$$
\operatorname{tr} \mathbf{F}_{2}\left(K^{*}\right)=F_{2}\left(K^{*}\right)
$$

We can seek for $\mathbf{F}_{2}\left(K^{*}\right)$ in the following form:

$$
\mathbf{F}_{2}\left(K^{*}\right)=a\left(K^{*}\right) \mathbf{I}_{n}+\frac{b\left(K^{*}\right)}{K^{* 2}} \mathbf{K}^{*} \otimes \mathbf{K}^{*}
$$

which is the most general form of a second-order tensor depending on the vector $\mathbf{K}^{*}$. Taking the trace gives

$$
F_{2}\left(K^{*}\right)=n a\left(K^{*}\right)+b\left(K^{*}\right)
$$

A second relation is required to compute the unknown functions $a\left(K^{*}\right)$ and $b\left(K^{*}\right)$. For this purpose, we may write the divergence of (63) with respect to $\mathbf{K}^{*}$ :

$$
\begin{aligned}
\nabla_{\mathbf{K}^{*}} \cdot \mathbf{F}_{2}\left(K^{*}\right) & =-2 i \sigma \int_{\Omega} \exp (-i \mathbf{K} \cdot \tilde{\mathbf{r}}) \nabla_{\tilde{\mathbf{r}}} w(\tilde{r}) d \tilde{\mathbf{r}} \\
& =2 \widehat{w}\left(K^{*}\right) \mathbf{K}^{*}
\end{aligned}
$$

(we made use of (16)). On the other hand, the $\mathbf{K}^{*}$-divergence of the right-hand side of (92) gives

$$
\begin{gathered}
\nabla_{\mathbf{K}^{*}} \cdot\left[a\left(K^{*}\right) \mathbf{I}_{n}\right]=\frac{\partial a\left(K^{*}\right) \delta_{i j}}{\partial K_{j}^{*}} \mathbf{e}_{i}=\frac{a^{\prime}\left(K^{*}\right)}{K^{*}} \mathbf{K}^{*} \\
\nabla_{\mathbf{K}^{*}} \cdot\left[\frac{b\left(K^{*}\right)}{K^{* 2}} \mathbf{K}^{*} \otimes \mathbf{K}^{*}\right]=\left[\frac{b^{\prime}\left(K^{*}\right)}{K^{*}}+(n-1) \frac{b\left(K^{*}\right)}{K^{* 2}}\right] \mathbf{K}^{*}
\end{gathered}
$$

Substituting into (92), we get

$$
2 K^{*} \widehat{w}\left(K^{*}\right)=a^{\prime}\left(K^{*}\right)+b^{\prime}\left(K^{*}\right)+(n-1) \frac{b\left(K^{*}\right)}{K^{*}}
$$


Differentiating (93) with respect to $K^{*}$ and re-arranging with (96) gives a firstorder linear ordinary differential equation in $b\left(K^{*}\right)$ :

$$
K^{*} b^{\prime}\left(K^{*}\right)+n b\left(K^{*}\right)=2 K^{* 2} \widehat{w}\left(K^{*}\right)
$$

This equation should be solved with the boundary condition $b(0)=0$, since $\mathbf{F}_{2}(0)$ obviously vanishes. The solution is

$$
b\left(K^{*}\right)=\frac{2}{K^{* n}} \int_{0}^{K^{*}} \kappa^{n+1} \widehat{w}(\kappa) d \kappa
$$

Rearranging (92) with (93) and (98) finally yields

$$
\mathbf{F}_{2}\left(K^{*}\right)=\frac{1}{n}\left[F_{2}\left(K^{*}\right)-b\left(K^{*}\right)\right] \mathbf{I}_{n}+\frac{b\left(K^{*}\right)}{K^{* 2}} \mathbf{K}^{*} \otimes \mathbf{K}^{*}
$$

according to (64). Now, for the Gaussian kernel $b\left(K^{*}\right)$ is easy to compute. As a consequence, the function $F_{2}^{M G}$ defined by (67) is given by the following formulae, according to the space dimension:

$$
\begin{aligned}
& n=2: F_{2}^{M G}\left(K^{*}\right)=4\left[1+\frac{2}{K^{* 2}}-2\left(1+\frac{1}{K^{* 2}}\right) \exp \left(-\frac{K^{* 2}}{2}\right)\right] \\
& n=3: F_{2}^{M G}\left(K^{*}\right)=\frac{10}{3}\left[1+\frac{3 \sqrt{2 \pi}}{K^{* 3}} \operatorname{erf}\left(\frac{\sqrt{2}}{2} K^{*}\right)-3\left(1+\frac{2}{K^{* 2}}\right) \exp \left(-\frac{K^{* 2}}{2}\right)\right]
\end{aligned}
$$

We will finally investigate the ratio $F_{2}\left(K^{*}\right) / F_{1}\left(K^{*}\right)$ at small wavenumbers. For this purpose, we proceed to a Taylor expansion of $\exp (-i \mathbf{K} \cdot \tilde{\mathbf{r}})$ in the definition of $\widehat{w}$ and use the normalizing property of $w$ :

$$
\begin{aligned}
\widehat{w}\left(K^{*}\right) & =\int_{\Omega}\left[1-i \mathbf{K} \cdot \tilde{\mathbf{r}}-\frac{1}{2}(\mathbf{K} \cdot \tilde{\mathbf{r}})^{2}+O\left(K^{3} \tilde{r}^{3}\right)\right] w(\tilde{r}) d \tilde{\mathbf{r}} \\
& =1-i \mathbf{K} \cdot \int_{\Omega} \tilde{\mathbf{r}} w(\tilde{r}) d \tilde{\mathbf{r}}-\frac{1}{2} \mathbf{K}^{T}\left[\int_{\Omega} \tilde{\mathbf{r}} \otimes \tilde{\mathbf{r}} w(\tilde{r}) d \tilde{\mathbf{r}}\right] \mathbf{K}+O\left(K^{* 3}\right)
\end{aligned}
$$

Now, the integral of $\tilde{\mathbf{r}} w(\tilde{r})$ vanishes since it is an odd function (the same property occurs with the integral of the third order). As a consequence, the first derivative of the Fourier transform of the kernel vanishes at the origin, i.e. $\widehat{w}^{\prime}(0)=0$, in agreement with Figure 1. As for the last integral in (101), it is an isotropic tensor with trace equal to $n \sigma^{2}$ (according to (2)), thus it is equal to $\sigma^{2} \mathbf{I}_{n}$, and

$$
\widehat{w}\left(K^{*}\right)=1-\frac{K^{* 2}}{2}+O\left(K^{* 4}\right)
$$

We then can write

$$
F_{1}\left(K^{*}\right)=K^{* 2}-K^{* 4}+O\left(K^{* 6}\right)
$$


Next, the relation (90), along with $(102)$ and $F_{2}\left(K^{*}\right)=0$, leads to

$$
F_{2}\left(K^{*}\right)=K^{* 2}-\frac{K^{* 4}}{4}+O\left(K^{* 6}\right)
$$

We see that the functions $\widehat{w}, F_{1}$ and $F_{2}$ behave similarly at small wavenumbers, regardless of the kernel choice. Lastly, (103) and (104) show that $F_{2}\left(K^{*}\right) / F_{1}\left(K^{*}\right)$ tends towards 1 when $K^{*}$ approaches zero.

\subsection{Appendix 3}

The Fourier transform of the Gaussian and Wendland order 5 kernels were presented in Section 2.4. The B-Spline kernels are defined so that their Fourier transform in dimension $n=1$ is a power of a 'sinc' function. When seeking for their Fourier transforms in higher dimensions, the case of the third dimension is easier, since the volume integration element is $d \tilde{\mathbf{r}}=\tilde{r}^{2} \sin \theta d \tilde{r} d \theta d \varphi=-\tilde{r}^{2} d \tilde{r} d(\cos \theta) d \varphi$, and it is possible to integrate over $\cos \theta$, which simplifies the complex exponential $\exp (-i \mathbf{K} \cdot \tilde{\mathbf{r}})=\exp (-i K \tilde{r} \cos \theta)$. In dimension $n=2$, however, the computations are somewhat more complex and involve the zero-order Bessel function of the first kind, $J_{0}$. It is then necessary to know the antiderivatives of functions like $x^{p} J_{0}(x)$ for arbitrary integer $p$. The latter are given from the Bessel function $J_{1}$ and the Struve functions $H_{0}$ and $H_{1}$ (see Abramovic and Stegun, 1972).

From these premises, it is not difficult to prove that the Wendland kernel of order 5 (defined by eqn (39) has the following Fourier transforms, according to the working dimension $n$ :

$$
\begin{aligned}
& n=1: \widehat{w}\left(K^{*}\right)=\frac{45}{2 K^{+6}}\left(K^{+2}+\frac{1}{2} K^{+} \sin 2 K^{+}-2 \sin ^{2} K^{+}\right) \\
& n=2: \widehat{w}\left(K^{*}\right)=\frac{105}{4 K^{+6}}\left[\begin{array}{c}
6 K^{+2} J_{0}\left(2 K^{+}\right)-K^{+} J_{1}\left(2 K^{+}\right) \\
+3 \pi\left(K^{+2}-\frac{5}{4}\right) Y\left(2 K^{+}\right)
\end{array}\right] \\
& n=3: \widehat{w}\left(K^{*}\right)=\frac{315}{8 K^{+8}}\left[\left(12-2 K^{+2}\right) \cos 2 K^{+}+9 K^{+} \sin 2 K^{+}+8 K^{+2}-12\right]
\end{aligned}
$$

where (and hereafter) $K^{+} \doteqdot(h / \sigma) K^{*}=h K$, and

$$
Y(x) \doteqdot J_{1}(x) H_{0}(x)-J_{0}(x) H_{1}(x)
$$


From (34), we then get the function $F_{2}\left(K^{*}\right)$ as

$$
\begin{aligned}
& n=1: F_{2}\left(K^{*}\right)=\frac{15}{7}+\frac{15}{14 K^{+4}}\left(3-6 K^{+2}-3 \cos 2 K^{+}\right) \\
& n=2: F_{2}\left(K^{*}\right)=\frac{35}{18}-\frac{175}{6 K^{+4}}\left[\begin{array}{c}
K^{+2} J_{0}\left(2 K^{+}\right)-\frac{1}{2} K^{+} J_{1}\left(2 K^{+}\right) \\
+\frac{\pi}{2}\left(K^{+2}-\frac{3}{4}\right) Y\left(2 K^{+}\right)
\end{array}\right] \\
& n=3: F_{2}\left(K^{*}\right)=\frac{28}{15}+\frac{21}{K^{+6}}\left(2-2 K^{+2}-2 \cos 2 K^{+}-K^{+} \sin 2 K^{+}\right)
\end{aligned}
$$

On similar grounds, we can write the Fourier transforms of the B-Spline kernel of order 3 (defined by eqn (40) :

$$
\begin{aligned}
& n=1: \widehat{w}\left(K^{*}\right)=\left(\frac{2}{K^{+}} \sin \frac{K^{+}}{2}\right)^{4} \\
& n=2: \widehat{w}\left(K^{*}\right)=\frac{60}{7 K^{+2}}\left[\begin{array}{c}
-\frac{1}{K^{+}} J_{1}\left(2 K^{+}\right)+2 J_{0}\left(2 K^{+}\right) \\
+\frac{1}{K^{+}} J_{1}\left(K^{+}\right)-J_{0}\left(K^{+}\right) \\
-\frac{\pi}{2 K^{+2}}\left(\frac{3}{2}-2 K^{+2}\right) Y\left(2 K^{+}\right) \\
+\frac{\pi}{2 K^{+2}}\left(3-K^{+2}\right) Y\left(K^{+}\right)
\end{array}\right] \\
& n=3: \widehat{w}\left(K^{*}\right)=\frac{12}{K^{+2}}\left(\frac{2}{K^{+}} \sin \frac{K^{+}}{2}\right)^{4}\left(1-\frac{K^{+}}{2} \cot \frac{K^{+}}{2}\right)
\end{aligned}
$$

with the same notation as above for $K^{+}$. The corresponding $F_{2}$ functions are given by

$$
\begin{gathered}
n=1: F_{2}\left(K^{*}\right)=\frac{8}{3}\left[\begin{array}{c}
-\frac{2}{K^{+2}} \sin ^{4} \frac{K^{+}}{2}-\frac{4}{K^{+}} \sin ^{3} \frac{K^{+}}{2} \cos \frac{K^{+}}{2} \\
-\operatorname{Ci}\left(2 K^{+}\right)+\operatorname{Ci}\left(K^{+}\right)+\ln 2
\end{array}\right] \\
n=2: F_{2}\left(K^{*}\right)=\frac{1860}{343}\left[\begin{array}{c}
-2 J_{0}\left(2 K^{+}\right)+\frac{1}{K^{+}} J_{1}\left(2 K^{+}\right) \\
+J_{0}\left(K^{+}\right)-\frac{1}{K^{+}} J_{1}\left(K^{+}\right) \\
-\frac{\pi}{2}\left(2-\frac{1}{2 K^{+2}}\right) Y\left(2 K^{+}\right) \\
+\frac{\pi}{2}\left(1-\frac{1}{K^{+2}}\right) Y\left(K^{+}\right)+\frac{1}{3}
\end{array}\right] \\
n=3: F_{2}\left(K^{*}\right)=\frac{9}{5}\left[1-\left(\frac{2}{K^{+}} \sin \frac{K^{+}}{2}\right)^{4}\right]
\end{gathered}
$$

In the previous equations, $\mathrm{Ci}$ stands for the cosine integral. 
As for the B-Spline kernel of order 4 (eqn (42)), we have

$$
\begin{aligned}
& n=1: \widehat{w}\left(K^{*}\right)=\left(\frac{2}{K^{+}} \sin \frac{K^{+}}{2}\right)^{5} \\
& n=2: \widehat{w}\left(K^{*}\right)=\frac{3840}{1199 K^{+2}}\left[\begin{array}{c}
5\left(\frac{25}{16}-\frac{2}{K^{+2}}\right) J_{0}\left(\frac{5 K^{+}}{2}\right)+\frac{1}{K^{+}}\left(\frac{8}{K^{+2}}-\frac{25}{8}\right) J_{1}\left(\frac{5 K^{+}}{2}\right) \\
+9\left(\frac{2}{K^{+2}}-\frac{9}{16}\right) J_{0}\left(\frac{3 K^{+}}{2}\right)+\frac{3}{K^{+}}\left(\frac{9}{8}-\frac{8}{K^{+2}}\right) J_{1}\left(\frac{3 K^{+}}{2}\right) \\
+\left(\frac{1}{8}-\frac{4}{K^{+2}}\right) J_{0}\left(\frac{K^{+}}{2}\right)+\frac{1}{K^{+}}\left(\frac{16}{K^{+2}}-\frac{1}{4}\right) J_{1}\left(\frac{K^{+}}{2}\right) \\
+\frac{5 \pi}{32}\left(25-\frac{36}{K^{+2}}\right) Y\left(\frac{5 K^{+}}{2}\right) \\
+\frac{81 \pi}{32}\left(\frac{4}{K^{+2}}-1\right) Y\left(\frac{3 K^{+}}{2}\right) \\
+\frac{\pi}{16}\left(1-\frac{36}{K^{+2}}\right) Y\left(\frac{K^{+}}{2}\right)
\end{array}\right] \\
& n=3: \widehat{w}\left(K^{*}\right)=\frac{12}{K^{+2}}\left(\frac{2}{K^{+}} \sin \frac{K^{+}}{2}\right)^{5}\left(1-\frac{K^{+}}{2} \cot \frac{K^{+}}{2}\right)
\end{aligned}
$$

and

$$
\begin{aligned}
& -\frac{20}{9 K^{+2}}\left(\frac{4}{K^{+}} \sin \frac{K^{+}}{2}+5 \cos \frac{K^{+}}{2}\right) \sin ^{4} \frac{K^{+}}{2} \\
& n=1: F_{2}\left(K^{*}\right)=\quad+\frac{50}{9 K^{+}}\left(5 \sin ^{2} \frac{K^{+}}{2}-4\right) \sin ^{3} \frac{K^{+}}{2} \\
& +\frac{25}{24}\left(-\frac{1}{3} \mathrm{Ci} \frac{K^{+}}{2}+\frac{9}{2} \mathrm{Ci} \frac{3 K^{+}}{2}-\frac{25}{6} \mathrm{Ci} \frac{5 K^{+}}{2}-\frac{9}{2} \ln 3+\frac{25}{6} \ln 5\right) \\
& {\left[\frac{5}{2}\left(\frac{1}{K^{+2}}-\frac{25}{8}\right) J_{0}\left(\frac{5 K^{+}}{2}\right)+\frac{1}{2 K^{+}}\left(\frac{25}{4}-\frac{4}{K^{+2}}\right) J_{1}\left(\frac{5 K^{+}}{2}\right)\right]} \\
& +\frac{9}{2}\left(\frac{9}{8}-\frac{1}{K^{+2}}\right) J_{0}\left(\frac{3 K^{+}}{2}\right)+\frac{3}{2 K^{+}}\left(\frac{4}{K^{+2}}-\frac{9}{4}\right) J_{1}\left(\frac{3 K^{+}}{2}\right) \\
& n=2: F_{2}\left(K^{*}\right)=\frac{2342160}{914837}+\left(\frac{1}{K^{+2}}-\frac{1}{8}\right) J_{0}\left(\frac{K^{+}}{2}\right)+\frac{1}{K^{+}}\left(\frac{1}{4}-\frac{4}{K^{+2}}\right) J_{1}\left(\frac{K^{+}}{2}\right) \\
& +\frac{5 \pi}{8}\left(\frac{3}{K^{+2}}-\frac{25}{4}\right) Y\left(\frac{5 K^{+}}{2}\right) \\
& +\frac{27 \pi}{8}\left(\frac{3}{4}-\frac{1}{K^{+2}}\right) Y\left(\frac{3 K^{+}}{2}\right) \\
& +\frac{\pi}{4}\left(\frac{3}{K^{+2}}-\frac{1}{4}\right) Y\left(\frac{K^{+}}{2}\right)+\frac{2760}{3840} \\
& n=3: F_{2}\left(K^{*}\right)=\frac{46}{25}\left[1-\left(\frac{2}{K^{+}} \sin \frac{K^{+}}{2}\right)^{5}\right]
\end{aligned}
$$

Finally, the B-Spline kernel of order 5 (defined by eqn (42)), we found the following 
Fourier transforms:

$$
\begin{aligned}
& n=1: \widehat{w}\left(K^{*}\right)=\left(\frac{2}{K^{+}} \sin \frac{K^{+}}{2}\right)^{6} \\
& n=2: \widehat{w}\left(K^{*}\right)=\frac{105}{239 K^{+2}}\left[\begin{array}{c}
9\left(9-\frac{17}{K^{+2}}\right) J_{0}\left(3 K^{+}\right)+\frac{3}{K^{+}}\left(\frac{19}{K^{+2}}-9\right) J_{1}\left(3 K^{+}\right) \\
+16\left(\frac{17}{K^{+2}}-4\right) J_{0}\left(2 K^{+}\right)+\frac{8}{K^{+}}\left(4-\frac{19}{K^{+2}}\right) J_{1}\left(2 K^{+}\right) \\
+5\left(1-\frac{17}{K^{+2}}\right) J_{0}\left(K^{+}\right)+\frac{5}{K^{+}}\left(\frac{19}{K^{+2}}-1\right) J_{1}\left(K^{+}\right) \\
+\frac{9 \pi}{2}\left(\frac{1}{K^{+2}}-3\right)\left(\frac{5}{K^{+2}}-3\right) Y\left(3 K^{+}\right) \\
-2 \pi\left(\frac{3}{K^{+2}}-4\right)\left(\frac{15}{K^{+2}}-4\right) Y\left(2 K^{+}\right) \\
+\frac{5 \pi}{2}\left(\frac{3}{K^{+2}}-1\right)\left(\frac{15}{K^{+2}}-1\right) Y\left(K^{+}\right) \\
n=3: \widehat{w}\left(K^{*}\right)=\frac{12}{K^{+2}}\left(\frac{2}{K^{+}} \sin \frac{K^{+}}{2}\right)\left(1-\frac{K^{+}}{2} \cot \frac{K^{+}}{2}\right)
\end{array}\right]
\end{aligned}
$$

while its $F_{2}$ functions are given by

$$
\begin{aligned}
& -\left(\frac{2}{K^{+}}\right)^{4} \sin ^{6} \frac{K^{+}}{2} \\
& n=1: F_{2}\left(K^{*}\right)=-\left(\frac{2}{K^{+}}\right)^{3} \sin ^{4} \frac{K^{+}}{2}\left(\frac{3}{2} K^{+} \cos K^{+}+\sin K^{+}+K^{+}\right) \\
& -\frac{2}{K^{+}} \sin ^{2} \frac{K^{+}}{2} \sin K^{+}\left(9 \cos K^{+}+1\right)-\frac{27}{4} \mathrm{Ci}\left(3 K^{+}\right) \\
& +8 \mathrm{Ci}\left(2 K^{+}\right)-\frac{5}{4} \mathrm{Ci}\left(K^{+}\right)+\frac{27}{4} \ln 3-8 \ln 2 \\
& n=2: F_{2}\left(K^{*}\right)=96985 \text { 228484 }\left[\begin{array}{c}
9\left(\frac{5}{K^{+2}}-9\right) J_{0}\left(3 K^{+}\right)+\frac{3}{K^{+}}\left(9-\frac{7}{K^{+2}}\right) J_{1}\left(3 K^{+}\right) \\
+16\left(4-\frac{5}{K^{+2}}\right) J_{0}\left(2 K^{+}\right)+\frac{8}{K^{+}}\left(\frac{7}{K^{+2}}-4\right) J_{1}\left(2 K^{+}\right) \\
+5\left(\frac{5}{K^{+2}}-1\right) J_{0}\left(K^{+}\right)+\frac{5}{K^{+}}\left(1-\frac{7}{K^{+2}}\right) J_{1}\left(K^{+}\right) \\
-\frac{9 \pi}{2}\left(\frac{1}{K^{+2}}-3\right)^{2} Y\left(3 K^{+}\right) \\
+2 \pi\left(\frac{3}{K^{+2}}-4\right)^{2} Y\left(2 K^{+}\right) \\
-\frac{5 \pi}{2}\left(\frac{3}{K^{+2}}-1\right)^{2} Y\left(K^{+}\right)+\frac{22}{5}
\end{array}\right] \\
& n=3: F_{2}\left(K^{*}\right)=\frac{28}{15}\left[1-\left(\frac{2}{K^{+}} \sin \frac{K^{+}}{2}\right)^{6}\right]
\end{aligned}
$$

The first and lines of eqns (108), (109) and (111) are consistent with Dehnen and Aly (2012), although they used the kernel support size $H$ to make the wavenumber adimensional. 


\subsection{References}

Abramowitz, M., Stegun, I.A., Handbook of Mathematical Functions with Formulas, Graphs, and Mathematical Tables, Dover Publications, New York, 1972 (10 ${ }^{\text {th }}$ edition).

Adami, S., Hu, X.Y, Adams, N.A. (2013), A transport-velocity formulation for Smoothed Particle Hydrodynamics, J. Comput. Phys. (accepted).

Amicarelli, A., Marongiu, J.-C., Leboeuf, F., Leduc, J., Fang, L., Caro, J. (2011), SPH truncation error in estimating a $3 D$ derivative, Int. J. Num. Meth. Eng. $87(7): 677-700$.

Antuono, M., Colagrossi, A., Marrone, S. (2012), On the use of numerical diffusive terms in weakly-compressible SPH schemes, Proc. 7th SPHERIC Int. workshop, Prato (Italy), pp. 200-207.

Archambeau, F., Mechitoua, N., Sakiz, M. (2004), Code_Saturne: a Finite Volume Code for urbulent Flows, Int. J. Finite Volumes 1, http://www.ijfv.fr/memo/JOURNAL/PRINTS/saturne.pdf.

Balsara, D.S. (1995), Von Neumann stability analysis of Smoothed Particle Hydrodynamics - Suggestions for optimal algorithms, J. Comput. Phys. 121:357-372.

Basa, M., Quinlan, N.J., Lastiwka, M. (2009), Robustness and accuracy of SPH formulations for viscous flow, Int. J. Numer. Meth. Fluids 60:1127-1148.

Bonet, J., Lok, T.-S.L. (1999), Variational and momentum preservation aspects of Smoothed Particle Hydrodynamics formulations, Comput. Meth. Appl. Mech. Eng. 180:97-115.

Campbell, J.C., Vignjevic, R., Libersky L. (2000), A contact algorithm for Smoothed Particle Hydrodynamics, Comput. Meth. Appl.Mech. Eng. 184:49-65.

Cherfils, J.-M. (2011), Développements et applications de la méthode SPH aux écoulements visqueux à surface libre, Ph.D. Thesis, Université du Havre (in French).

Colagrossi, A., Antuono, M., Souto-Iglesias, A., Le Touzé, D., Izaguirre-Alza, P. (2010), Theoretical analysis of SPH in simulating free-surface viscous flows, Proc. 5th SPHERIC Int. workshop, Manchester (UK), pp. 138-145.

Colagrossi, A., Bouscasse, B., Antuono, M., Marrone, S. (2012), Particle packing algorithm for SPH schemes, Comput. Phys. Comm. 183:1641-1653.

De Leffe, M. (2011), Modélisation d'écoulements visqueux par méthode SPH en vue d'application à l'hydrodynamique navale, Ph.D. Thesis, Ecole Centrale de Nantes (in French).

Dehnen, W., Aly, H. (2012), Improving convergence in smoothed particle hydrody- 
namics simulations without pairing instability, Mon. Not. R. Astron. Soc. 000:1-15.

Fatehi, R., Manzari, F.T. (2011), Error estimation in smoothed particle hydrodynamics and a new scheme for second derivatives, Comput. Math. Appl. 61:482-498.

Ferrand, M., Laurence, D., Rogers, B., Violeau, D., Kassiotis, C. (2012), Unified semi-analytical wall boundary conditions for inviscid, laminar or turbulent flows in the meshless SPH method, Int. J. Num. Meth. Fluids DOI: 10.1002/fld.3666.

Ferrari, A., Dumbser, M., Toro, E.F., Armanini, A. (2009), A new 3D parallel SPH scheme for free surface flows, Comput. Fluids 38:1203-1217.

Ghia, U., Ghia, K., Shin, C.A. (1982), High-Resolutions for incompressible flow using the Navier-Stokes equations and a multigrid method, J. Comput. Phys. 48:387411.

Hu, X.Y., Adams, N.A. (2006), A multiphase SPH method for macroscopic and mesoscopic flows, J. Comput. Phys. 213:844-861.

Lee, E.-S., Moulinec, C., Xu, R., Violeau, D., Laurence, D., Stansby, P. (2008), Comparisons of weakly compressible and truly incompressible SPH algorithms for $2 D$ flows, J. Comput. Phys. 227(18):8417-8436.

Mayrhofer, A., Rogers, B.D., Violeau, D., Ferrand, M. (2013), Investigation of wall bounded flows using SPH and the unified semi-analytical wall boundary conditions, Comput. Phys. Com. (in press).

Monaghan, J.J. (1985a), Extrapolating B-Splines for interpolation, J. Comput. Phys. 60:253-262.

Monaghan, J.J. (1992), Smoothed particle hydrodynamics, Ann. Rev. Astr. Astroph. 30:543-574.

Monaghan, J.J. (1994), Simulating free surface flows with SPH, J. Comput. Phys. 110:399-406.

Monaghan, J.J. (2005), Smoothed Particle Hydrodynamics, Rep. Prog. Phys 68:1703-1759.

Monaghan, J.J. and Gingold, R.A. (1983), Shock simulation by the particle method SPH, J. Comput. Phys. 52(2):374-389.

Morris, J.P. (1996), Analysis of Smoothed Particle Hydrodynamics with applications, Ph.D. thesis, University of Monash, Melbourne (Australia).

Morris, J.P., Fox, P.J., Zhu, Y. (1997), Modelling low Reynolds number incompressible flows using SPH, J. Comput. Phys. 136:214-226.

Oger, G., Doring, M., Alessandrini, B., Ferrant, P. (2007), An improved SPH 
method: towards higher order convergence, J. Comput. Phys. 225:1472-1492.

Price, D.J. (2012), Smoothed particle hydrodynamics and magnetohydrodynamics, J. Comp. Phys. 231(3):759-794.

Quinlan, N.J., Basa, M., Lastiwka, M. (2006). Truncation error in mesh-free particle methods, Int. J. Num. Meth. Eng. 66:2064-2085.

Randles, P.W., Libersky, L.D. (1996), SPH: some recent improvements and applications, Comput. Meth. Applied Mech. Eng. 139:375-408.

Robinson, M. (2009), Turbulence and viscous mixing using Smoothed Particle Hydrodynamics, Ph.D. thesis, University of Monash, Melbourne (Australia).

Schwaiger, H.F. (2008), An implicit corrected SPH formulation for thermal diffusion with linear free-surface boundary condition, Int. J. Num. Meth. Eng. 75:647671.

Swegle, J.W., Hicks, D.L., Attaway, S.W. (1995), Smoothed Particle Hydrodynamics Stability Analysis, J. Comput. Phys. 116:123-134.

Vila, J.-P. (1999), On particle weighted methods and SPH, Math. Model Meth. Appl. Sci. 9:161-210.

Violeau, D. (2012), Fluid Mechanics and the SPH Method, Oxford University Press, Oxford.

Violeau, D., Issa, R. (2006), Numerical modelling of complex turbulent free surface flows with the SPH Lagrangian method: an overview, Int. J. Num. Meth. Fluids 53(2):277-304.

Wendland, H. (1995), Piecewise polynomial, positive definite and compactly supported radial functions of minimal degree, Adv. Comput. Math. 4:389-396. 


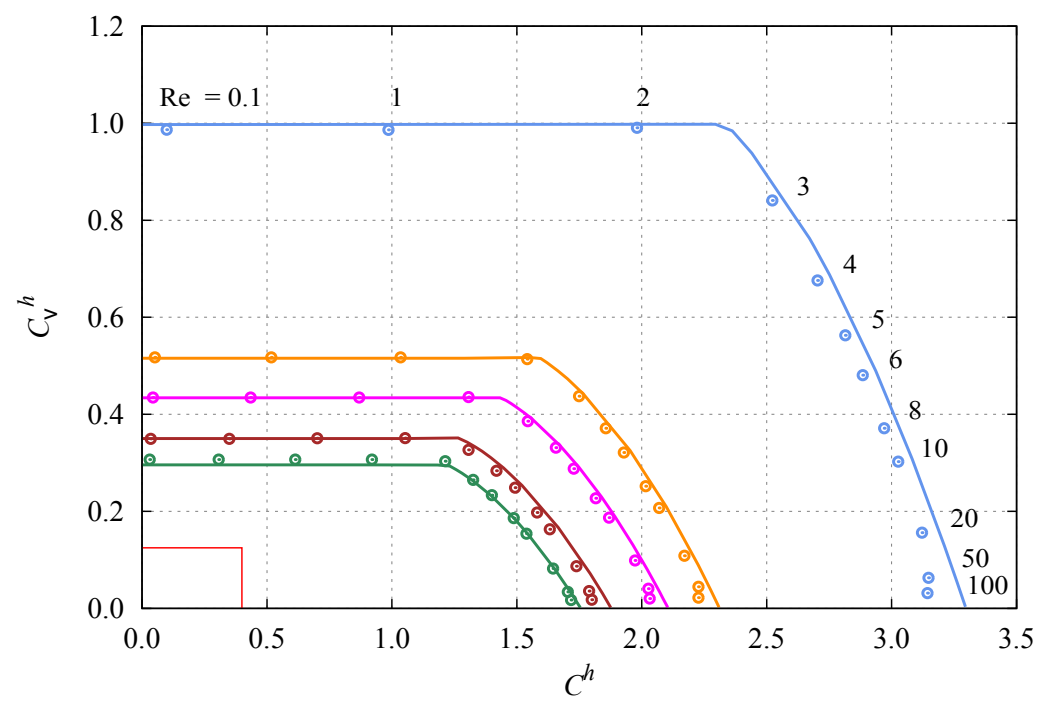

Figure 3: Stability domains in the $\left(C^{h}, C_{\nu}^{h}\right)$-plane for the various kernels presented here in dimension $n=2$. Solid lines: theory according to equation (33). Symbols: numerical results. The symbols are coloured according to the lines, with same colour nomenclature as in Fig. 1. The values of the $R e_{0}=C / C_{\nu}=C^{h} / C_{\nu}^{h}$ (see eqn (28)) are also given. The red line shows the traditional stability domain (46). 


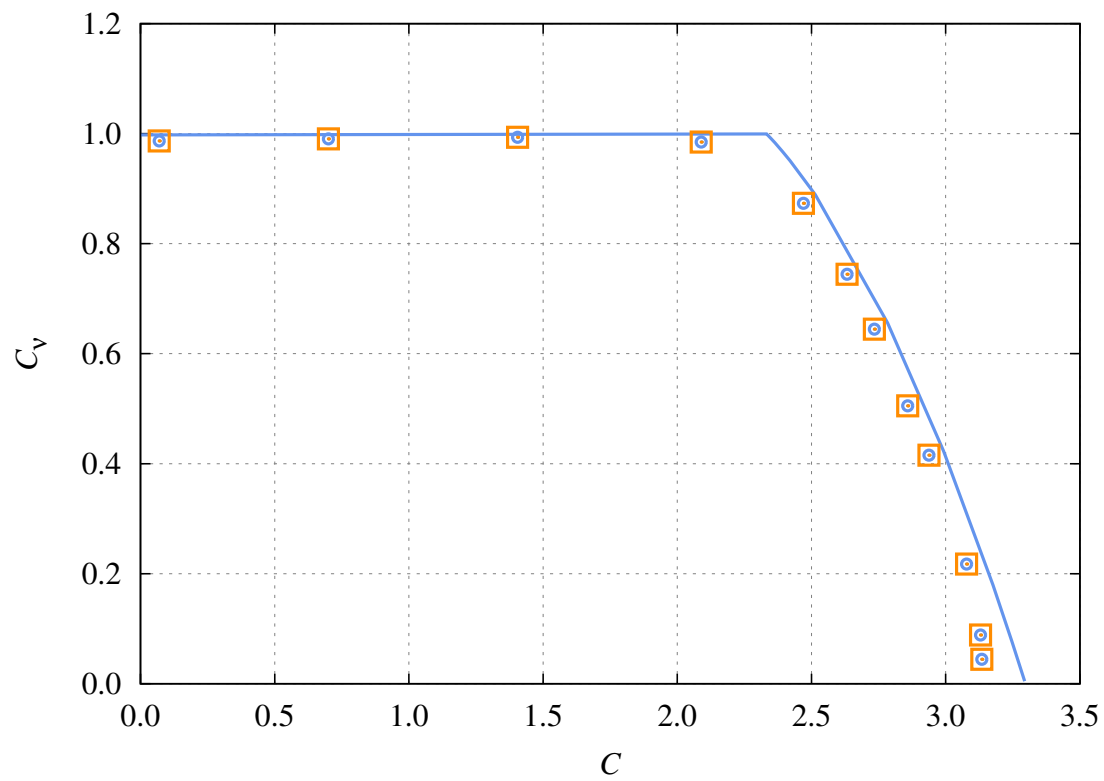

Figure 4: Stability domains in the $\left(C, C_{\nu}\right)$-plane for the Gaussian kernel in dimension $n=2$ for two estimations of the density. Solid line: theory according to equation (33). Circles: numerical results with the continuity equation (second line of (11)); squares: numerical results with the density interpolation (47) (here they match exactly the circles).

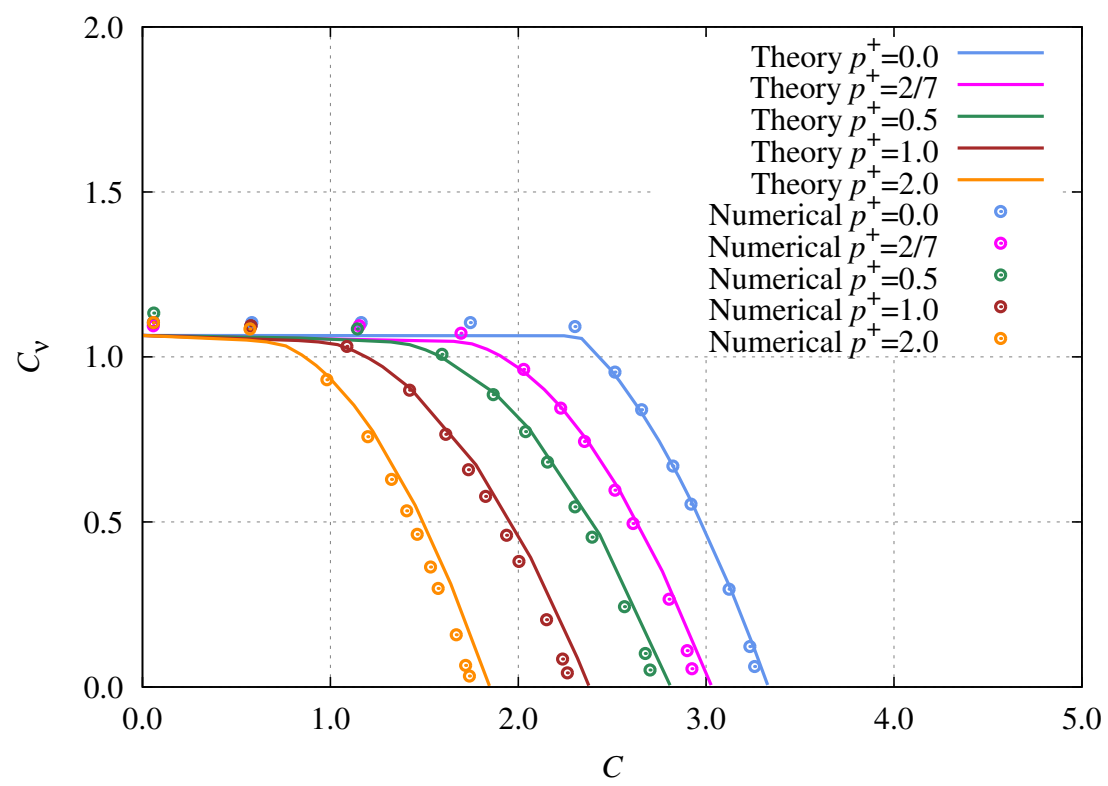

Figure 5: Stability domains in the $\left(C, C_{\nu}\right)$-plane for the Wendland kernel of order 5 in dimension $n=2$ for the operators $\mathbf{G}_{a}^{+}$and $D_{a}^{-}$and various values of $p^{+}$. Solid lines: theory according to equation (33). Symbols: numerical results. 


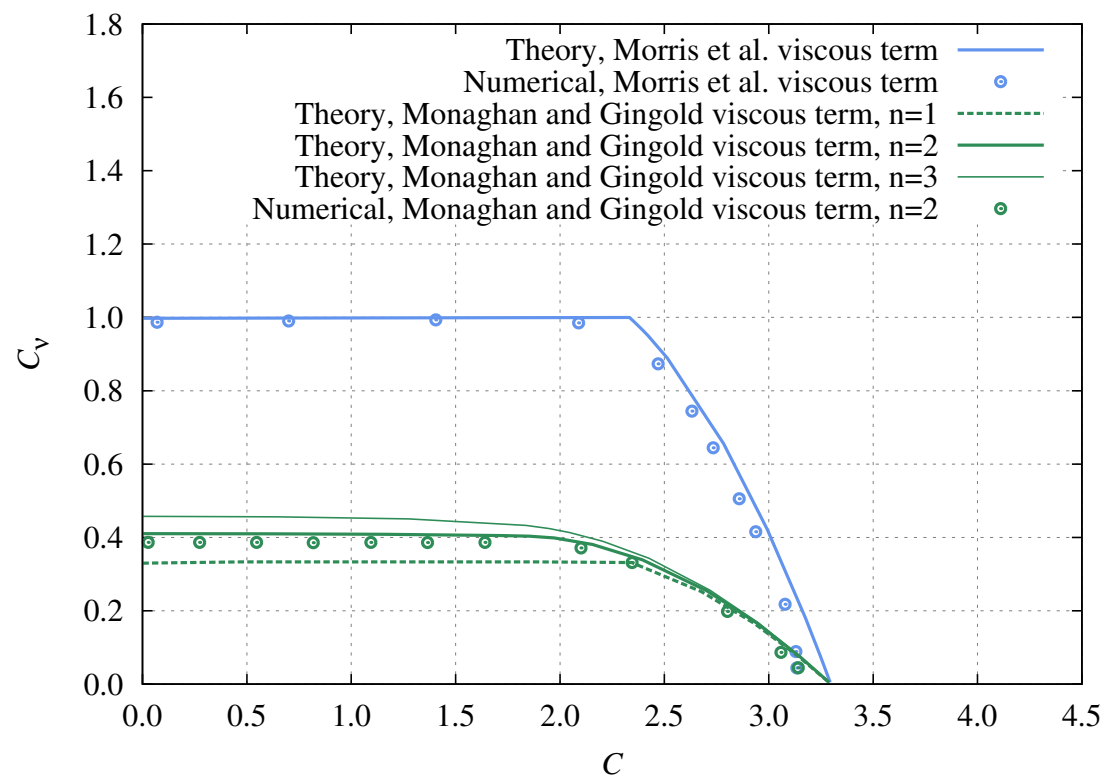

Figure 6: Stability domains in the $\left(C, C_{\nu}\right)$-plane for the Gaussian kernel and the SPH viscous forces as proposed by Morris et al. (1997, see eqn (10)) and Monaghan and Gingold (1983, eqn (62)). Solid lines: theory according to equation (33). Symbols: numerical results in dimension $n=2$.

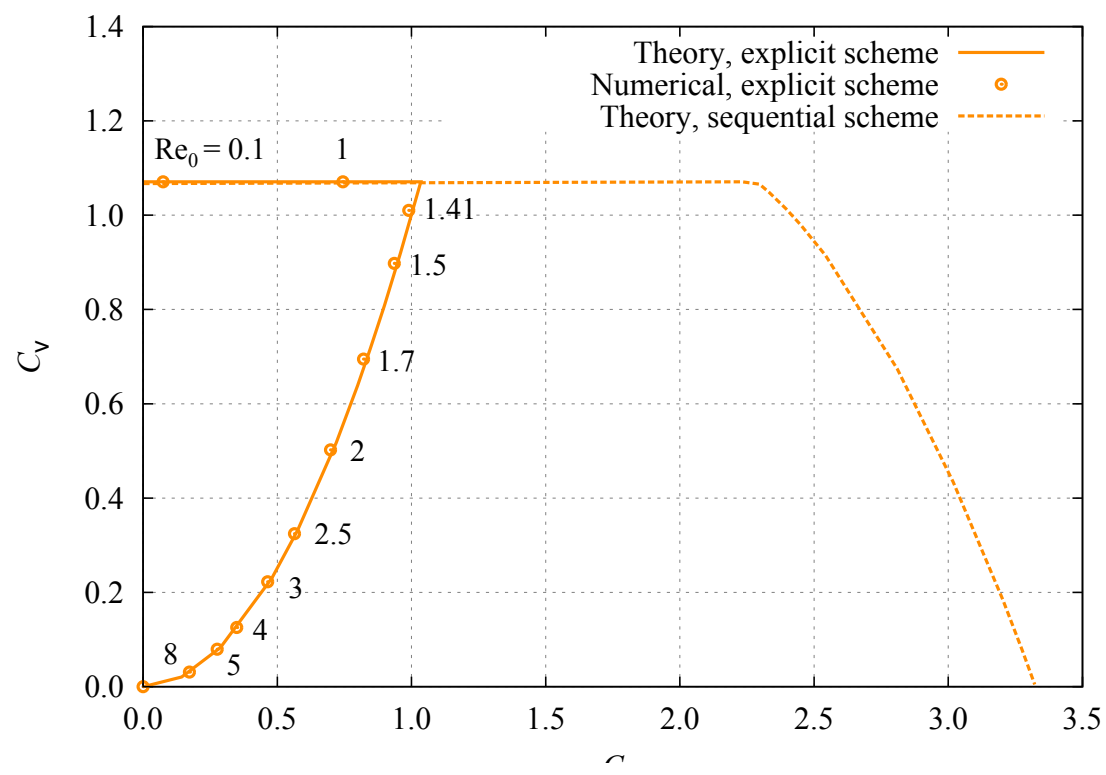

Figure 7: Stability domain in the $\left(C, C_{\nu}\right)$-plane for the B-Spline kernel of order 5 and the explicit time integration scheme of Section 3.2, in dimension $n=2$. Solid line: theory according to equation (73). Symbols: numerical results. The values of the $R e_{0}=C / C_{\nu}$ (see eqn (28)) are also given. For comparison, the stability domain from the sequential scheme is also plotted. 


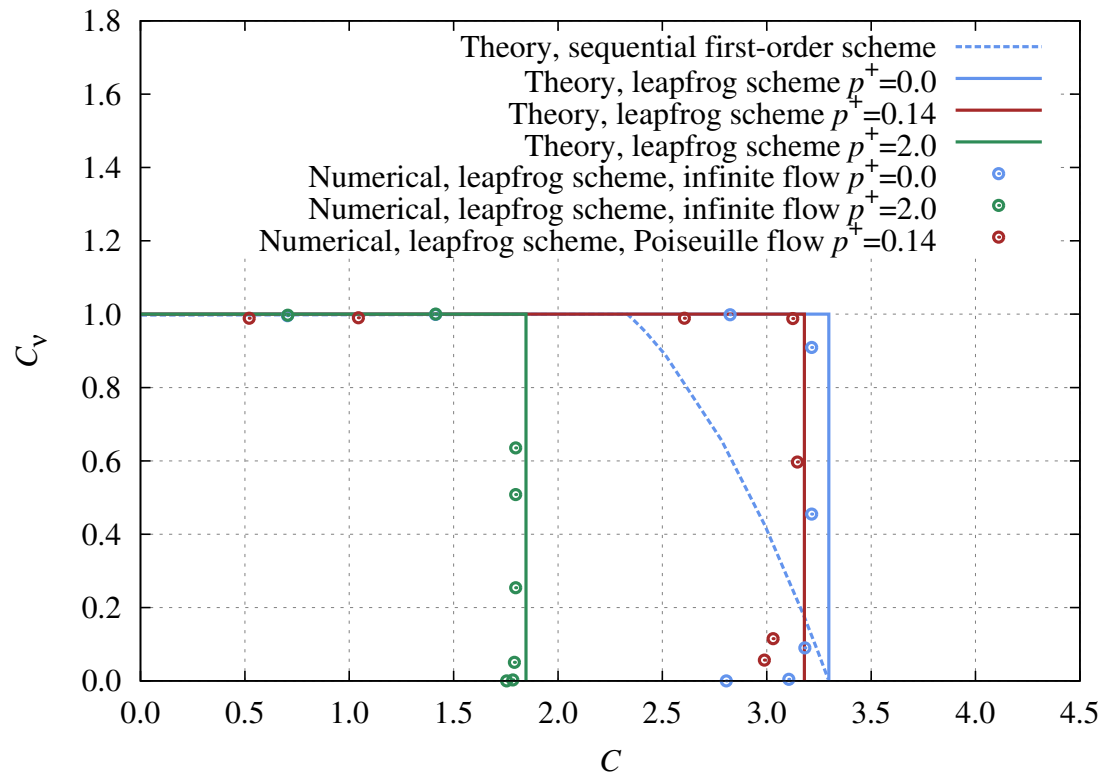

Figure 8: Stability domain in the $\left(C, C_{\nu}\right)$-plane for the Gaussian kernel and the leapfrog time integration scheme, in dimension $n=2$. Solid lines: theory according to equation (79). Symbols: numerical results. For comparison, the stability domain from the sequential first-order scheme is also plotted. We also display the Poiseuille flow case, discussed in Section 3.3.

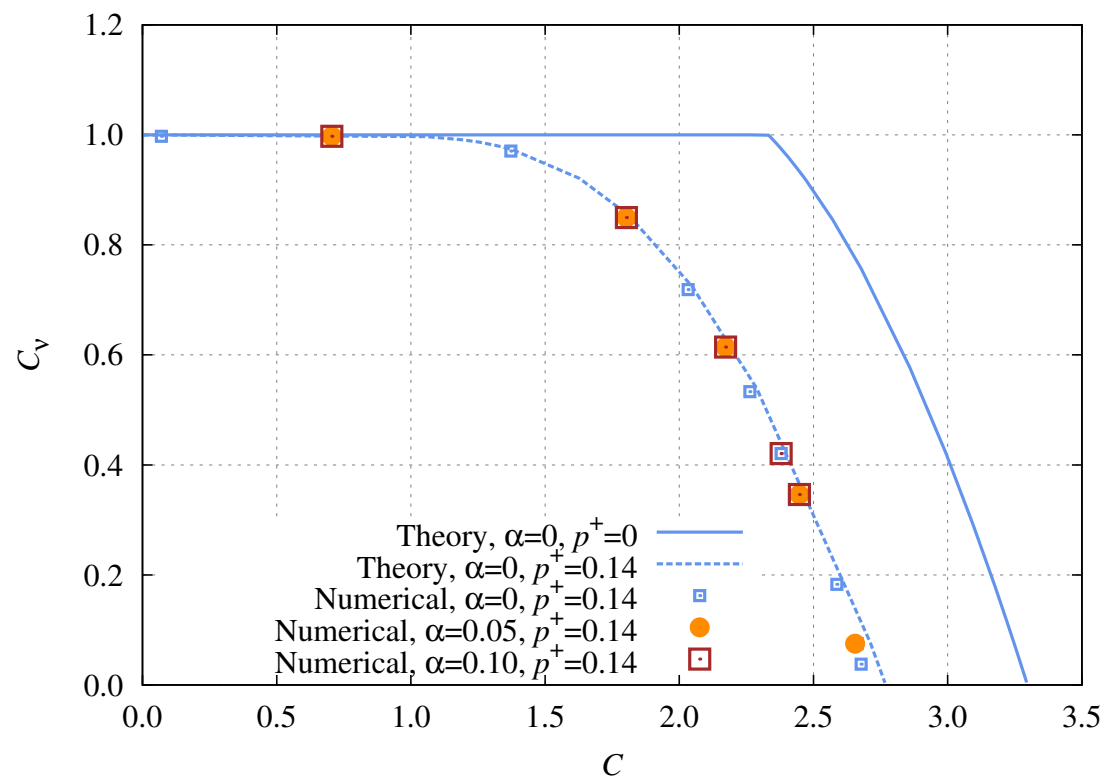

Figure 9: Stability domains in the $\left(C, C_{\nu}\right)$-plane for the Gaussian kernel and first-order sequential scheme. Lines: theory according to equation (33). Symbols: numerical results in dimension $n=2$. The blue symbols represent the infinite flow with constant velocity $(\alpha=0)$, the other colours a non-zero velocity gradient (sinusoidal flow (81)) 


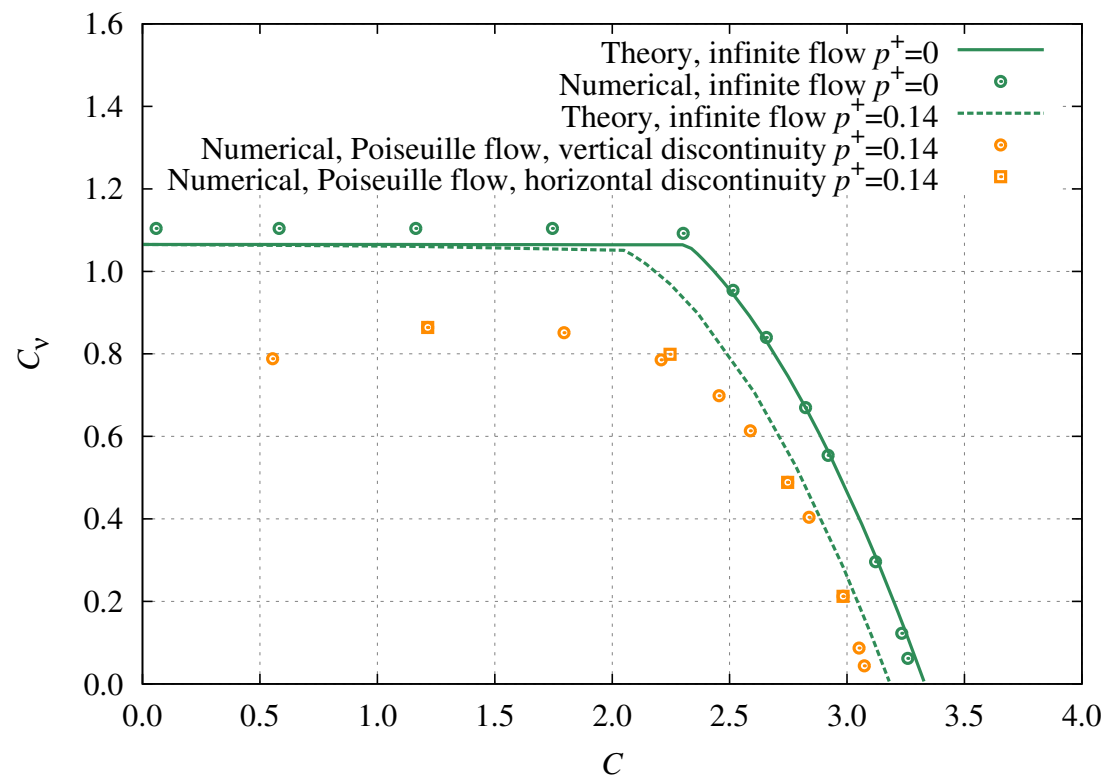

Figure 10: Stability domains in the $\left(C, C_{\nu}\right)$-plane for the Wendland kernel. Lines: theory according to equation (33). Symbols: numerical results in dimension $n=2$ with and without background pressure $p^{+}$. The green symbols represent the infinite flow with $p^{+}=0$, the orange ones the Poiseuille flow with $p^{+}=0.14$ for vertical (circles) and horizontal (squares) initial density discontinuity. 


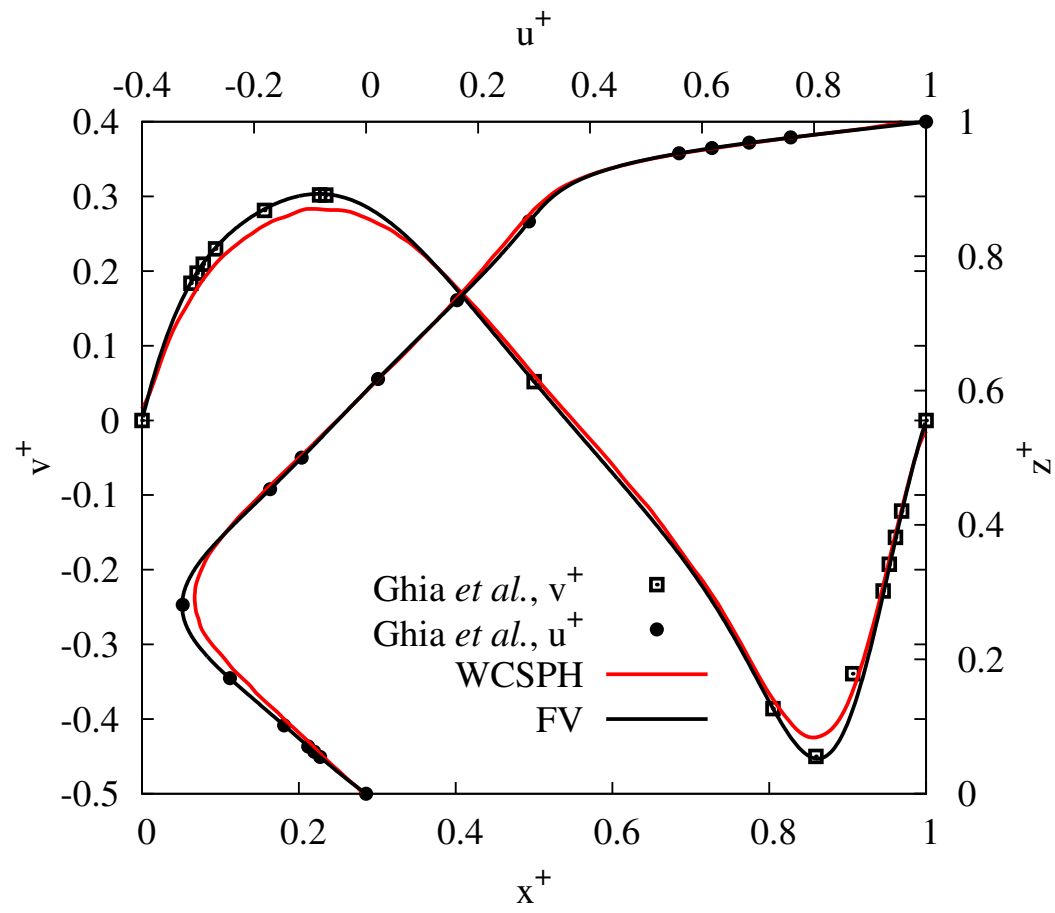

Figure 11: Velocity profiles along the two centerlines of the lid-driven cavity flow at $R e=400$ after convergence. Red line: SPH. Black line: Finite Volume (code_Saturne, see Archambeau et al., 2004). Symbols: simulations by Ghia et al. (1982).

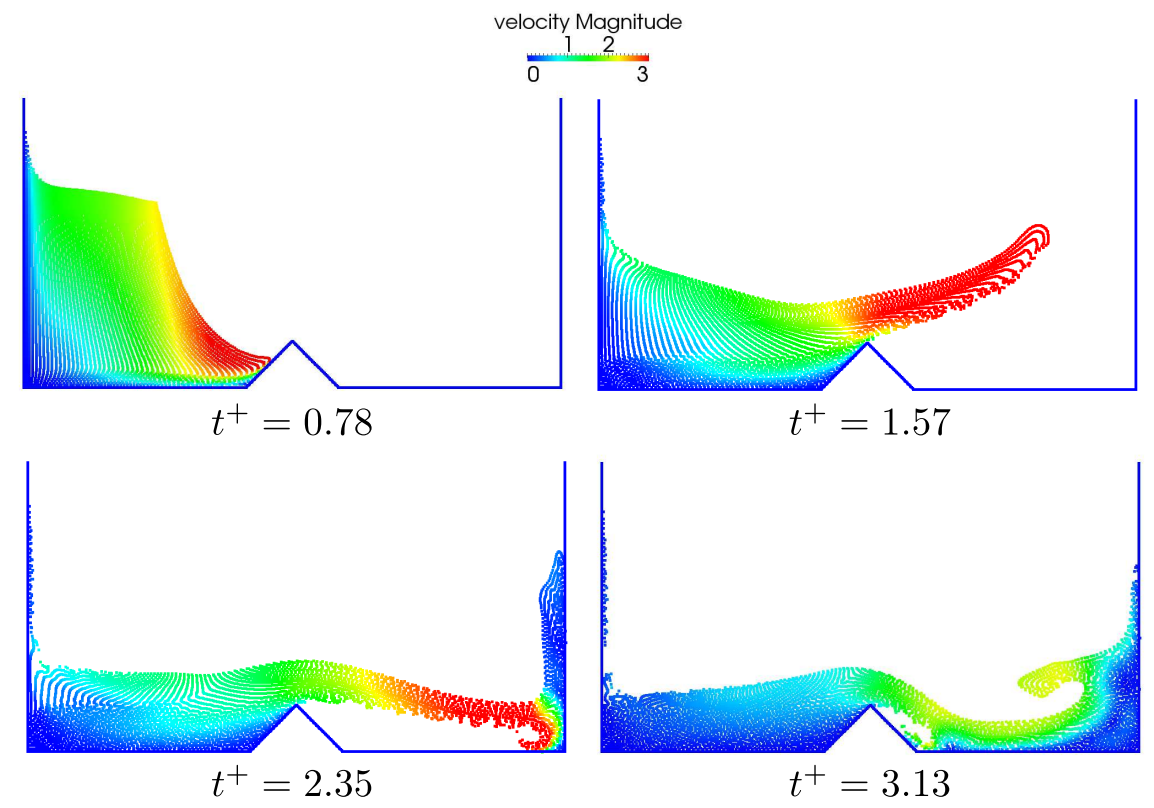

Figure 12: Snapshots of the water collapse case with a wedge modelled with SPH at four dimensionless instants. The contours show the velocity magnitude. 


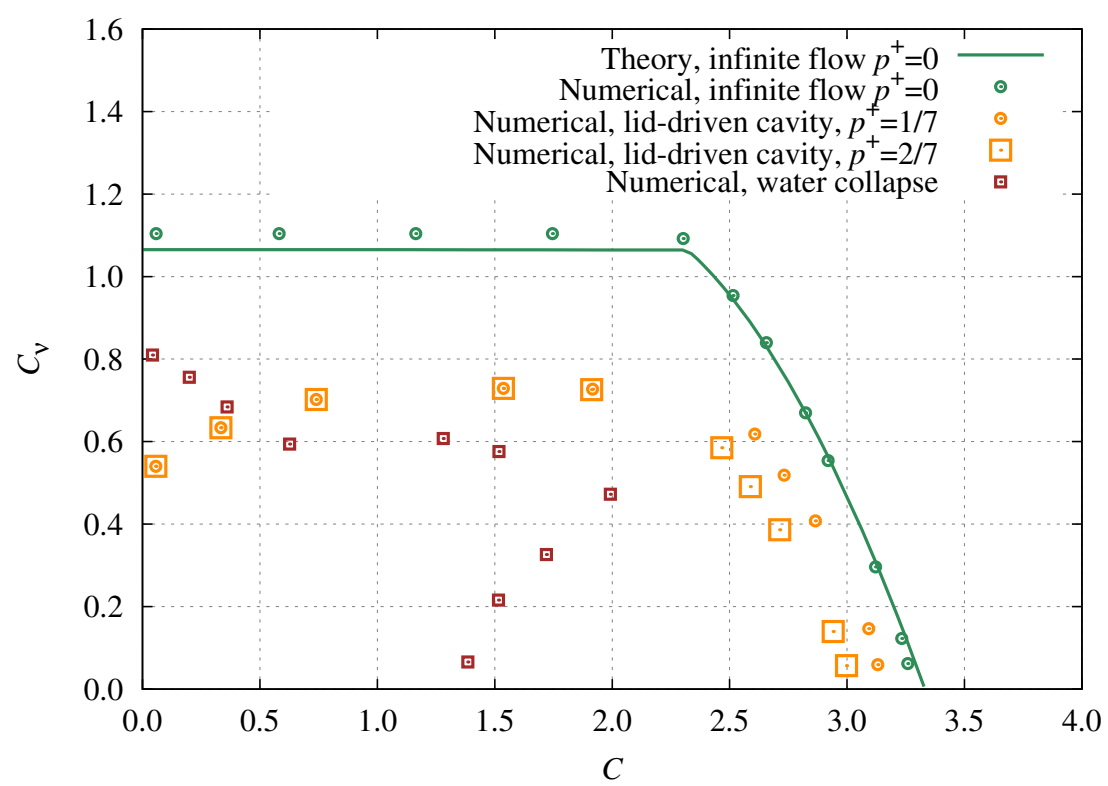

Figure 13: Stability domains in the $\left(C, C_{\nu}\right)$-plane for the Wendland kernel and first-order sequential scheme. Line: theory according to equation (33). Symbols: numerical results in dimension $n=2$ for various types of flows. 